\title{
Human DNA polymerase delta is a pentameric holoenzyme with a dimeric $\mathrm{p} 12$ subunit
}

\author{
Prashant Khandagale, Doureradjou Peroumal, Kodavati Manohar, Narottam Acharya (i)
}

\begin{abstract}
Human DNA polymerase delta (Pol $\delta$ ), a holoenzyme consisting of p125, p50, p68, and p12 subunits, plays an essential role in DNA replication, repair, and recombination. Herein, using multiple physicochemical and cellular approaches, we found that the p12 protein forms a dimer in solution. In vitro reconstitution and pull down of cellular Pol $\delta$ by tagged $p 12$ substantiate the pentameric nature of this critical holoenzyme. Furthermore, a consensus proliferating nuclear antigen (PCNA) interaction protein motif at the extreme carboxyl-terminal tail and a homodimerization domain at the amino terminus of the p12 subunit were identified. Mutational analyses of these motifs in p12 suggest that dimerization facilitates $\mathrm{p} 12$ binding to the interdomain connecting loop of PCNA. In addition, we observed that oligomerization of the smallest subunit of Pol $\delta$ is evolutionarily conserved as Cdm1 of Schizosaccharomyces pombe also dimerizes. Thus, we suggest that human Pol $\delta$ is a pentameric complex with a dimeric p12 subunit, and discuss implications of p12 dimerization in enzyme architecture and PCNA interaction during DNA replication.
\end{abstract}

DOI 10.26508/Isa.201900323 | Received 29 January 2019 | Revised 4 March 2019 | Accepted 11 March 2019 | Published online 18 March 2019

\section{Introduction}

Accurate and processive DNA synthesis by DNA polymerases (Pol) during chromosomal DNA replication is essential for lowering the rate of spontaneous mutations and suppressing carcinogenesis (Pavlov et al, 2006). Three essential DNA polymerases, namely, Pol $\alpha$, Pol$\delta$, and Pol $\varepsilon$, coordinate eukaryotic chromosomal DNA replication (Stillman, 2008; Kunkel \& Burgers, 2014, 2017; Burgers \& Kunkel, 2017). Based on biochemical and genetic studies, mostly those carried out in the budding yeast, it has been proposed that Pol $\alpha$ initiates DNA replication by synthesizing a short RNA-DNA primer, and is followed by loading of DNA clamp proliferating cell nuclear antigen (PCNA) by its loader replication factor C. Pol $\delta$ plays a major role in synthesizing "Okazaki fragments" in the lagging and initiating leading-strand DNA synthesis (Aria \& Yeeles, 2018). Pol $\varepsilon$ is involved in only leading-strand DNA synthesis (Acharya et al, 2011; Johnson et $\mathrm{al}$, 2015). In the absence of Polk, Pol $\delta$ also synthesizes the bulk of the leading strand. The mechanism of DNA replication in higher eukaryotes is yet to be deciphered; however, Pol $\delta$ replicates both the leading and lagging strands of the SV40 virus genome (Waga et al, 1994; Stillman, 2008). Irrespective of their different roles in DNA replication, these DNA polymerases possess certain commonalities such as the multi-subunit composition and signature sequences of a B-family DNA polymerase in the largest catalytic subunits (Tahirov et al, 2009; Kunkel \& Burgers, 2017).

Among the replicative DNA polymerases, the subunit composition of Pol $\delta$ varies between eukaryotes. Whereas Saccharomyces cerevisiae Pol $\delta$ consists of three subunits, Pol3, Pol31, and Pol32, Pol $\delta$ from Schizosaccharomyces pombe possesses four subunits, Pol3, Cdc1, Cdc27, and Cdm1 (Zuo et al, 2000; Acharya et al, 2011; Miyabe et al, 2011). The mammalian Pol $\delta$ holoenzyme consists of p125 as the catalytic subunit, the yeast homologue of Pol3, whereas p50, p68, and p12 are the structural subunits (Zhou et al, 2012). The accessory subunits p50 and p68 are the equivalents of Pol31/Cdc1 and Pol32/Cdc27 subunits, respectively. The p50/Pol31/Cdc1 subunit makes a connecting bridge between the catalytic subunit p125/ Pol3 and p68/Pol32/Cdc27 and is indispensable for cell viability. Although Pol32 is not essential for cell survival in S. cerevisiae, in its absence, cells exhibit sensitivity to both high and cold temperatures, and susceptibility to genotoxic stress (Johansson et al, 2004). Contrary to this, $\mathrm{Cdc} 27$ deletion strain of $\mathrm{S}$. pombe is not viable (Bermudez et al, 2002). The nonessential p12 subunit is the Cdm1 homologue and is absent in S. cerevisiae. Yeast two-hybrid and coimmunoprecipitation analyses suggested a dual interaction of p12 with p125 and p50; however, the modes of binding among these subunits are yet to be defined (Li et al, 2006). In vitro reconstitution has facilitated purification of four different subassemblies of hu-

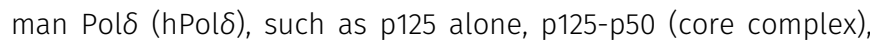
p125-p50-p68, and p125-p50-p68-p12 complexes, for biochemical studies. Reports also suggest that the subunit composition of hPol $\delta$ may alter in vivo with cellular response to DNA damage (Lee et al, 2012, 2014). Upon treatment of human cells with genotoxins such as UV, methyl methanesulfonate, hydroxyurea, and aphidicolin, the p12 subunit undergoes rapid degradation to result in a trimeric

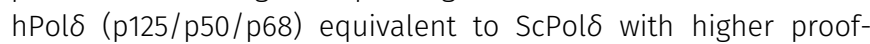
reading activity (Meng et al, 2010). Thus, p12 subunit seems to play a

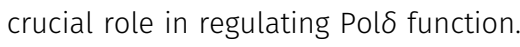

Laboratory of Genomic Instability and Diseases, Department of Infectious Disease Biology, Institute of Life Sciences, Bhubaneswar, India

Correspondence: narottam_acharya@ils.res.in 
The function of Pol $\delta$ as a processive DNA polymerase mostly depends upon its association with PCNA that acts as a sliding clamp (Krishna et al, 1994). The interaction of PCNA-binding proteins with PCNA gets mediated by a conserved PCNA-interacting protein motif (PIP-box) with a consensus sequence $Q-X-X-(M / L / I)-X-X-F F-(Y Y / L Y)$, where $x$ could be any amino acid (Haracska et al, 2005; Yoon et al, 2014). Previously, we have shown that all the three subunits of ScPol $\delta$ functionally interact with trimeric PCNA, an interaction mediated by their PIP motifs (Acharya et al, 2011). All three PIP boxes of ScPol $\delta$ are required to achieve higher processivity in vitro. Similarly, reports from hPold studies suggest that all the four subunits of hPol $\delta$ are involved in a multivalent interaction with PCNA and each of them regulates processive DNA synthesis by Pol $\delta$ (Wang et al, 2011). The PIP motifs have been identified in p68 and p50, but the same is yet to be mapped in p125 and p12 (Zhang et al, 1999; Lu et al, 2002). Studies based on far-Western and immunoprecipitation analyses revealed that the first 19 aa of p12 are involved in PCNA interaction, although the stretch lacks the canonical PIP box sequence (Li et al, 2006; Terai et al, 2013).

In this study, we have reinvestigated the interaction of $\mathrm{p} 12$ with other Pol $\delta$ subunits and PCNA. Our results indicate that the smallest subunit 12 exists as a dimer in solution to establish a dual interaction with both p125 and p50 subunits of Pol $\delta$. We have mapped the dimerization motif to the amino-terminal end and have identified a novel conserved PIP box in the C-terminal tail of p12. Importantly, the dimerization of p12 facilitates its interaction with PCNA. Based on our observations, we propose that hPol $\delta$ exists in a pentameric form in the cell in addition to other subassemblies, and we discuss the effect of p12 dimerization on PCNA binding with the various subassemblies.

\section{Results}

\section{Oligomerization of p12 subunit of human Pol $\delta$}

In several studies, hPol $\delta$ holoenzyme has been purified from either an insect cell line or bacterial expression system by using standard chromatography techniques for biochemical characterizations (Fazlieva et al, 2009; Rahmeh et al, 2012; Zhou et al, 2012). Although the purified Pol $\delta$ used in the various enzymatic assays contained all the four subunits p125, p50, p68, and p12, in most cases, the subunits were not in equimolar concentration. Especially, the smallest subunit p12 was comparatively in higher stoichiometry as compared with others (Fazlieva et al, 2009; Rahmeh et al, 2012). Even when we attempted to purify the hPol $\delta$ holoenzyme by using GSTaffinity beads, the band intensity of p12 protein was consistently higher than that of the other subunits (Fig S1). Such discrepancy in the composition of Pol $\delta$ could arise because of either the oligomeric status of p12 or multi-subunit p12 interaction with p125 and p50 or because of staining artifacts. Yeast two-hybrid assay and native PAGE analysis were carried out to examine the potential oligomerization status of the p12 subunit (Fig 1). p12 orf was fused in frame with both GAL4 activation (AD) and GAL4-binding domains (BD). Other hPol $\delta$ subunit orfs were fused with the GAL4-binding domain alone. The HFY7C yeast reporter strain harboring the AD-p12 plasmid was transformed with one of the BD-Pol $\delta$ subunit plasmids and selected on a Leu ${ }^{-}$Trp $^{-}$SDA plate. The interactions of p12 with other subunits of Pol $\delta$ in these transformants were analyzed by selecting them on plates lacking histidine. Growth on a His ${ }^{-}$plate demonstrates the interaction between the two fusion proteins as only the binding of two proteins makes it possible to form an intact GAL4 activator to confer HIS expression. As reported earlier, p12 interaction was observed with p125 and p50 but not with p68 (Fig 1A, sectors 1, 2, and 3) (Li et al, 2006). Surprisingly, the p12 subunit interacted with itself to give HIS expression (sector 4), whereas no growth was observed in the negative controls (sector 5 and 6). This result suggests that 12 makes specific multivalent interaction with itself and p125 and p50 subunits, but not with p68.

Furthermore, to ascertain oligomerization of p12, the protein was purified to near homogeneity from bacterial cells by using GSTaffinity column chromatography, GST-tag was cleaved off by Prescission protease, and analyzed by both native and SDScontaining PAGE. The predicted MW of $\mathrm{p} 12$ is $\sim 12 \mathrm{kD}$ with a $\mathrm{pl}$ of 6.3. p12 is known to possess abnormal migration in SDS-PAGE (Podust et al, 2002), and similarly, we observed the protein to migrate at 15-kD molecular weight size position (Fig 1B, lane 2). By taking advantage of native PAGE analysis where proteins resolve based on their charge and hydrodynamic size, we found that p12 migrated at a similar position with Carbonic anhydrase (CA) which is a protein of $30 \mathrm{kD}$ with pl 6.4 (lane 4 and 5). Thus, the similar migration of two proteins in non-denaturing PAGE indicates that both CA and 12 possess similar mass to charge ratio and the slower migration of 12 indicates that it is potentially a dimeric complex.

By taking advantage of isothermal calorimetry (ITC) technique, the oligomerization of p12 protein was examined (Fig 1C, i). p12 was placed both in the sample cell and in the syringe of the calorimeter. Twenty times $2 \mu \mathrm{l}$ of p12 was injected to p12-containing cell, and the binding of the ligand to the protein was analyzed by monitoring the change in heat. Upon p12-p12 interaction, the $\Delta \mathrm{H}, \Delta \mathrm{G}$, and $\mathrm{Kd}$ for the complex were estimated as $-1.82 \mathrm{kcal} / \mathrm{mol},-9.48 \mathrm{kcal} / \mathrm{mol}$, and $\sim 146 \mathrm{nM}$, respectively. The number of ligand-binding site as derived from the ITC analysis was found to be $\sim 0.6$, which is $~ 1: 1$ binding of p12 monomers. Thus, both in vivo and physiochemical studies indicate homodimerization of the p12 subunit.

\section{Cellular existence of oligomeric p12 in Pol $\delta$ complex and it's co-localization with PCNA}

HEK293 cells were transfected with GFP-p12 construct to establish p12 oligomerization in its cellular state. Such cells will harbor both native and GFP-tagged p12, and co-immunoprecipitation of Pol $\delta$ from such cell lysate will facilitate easy detection of oligomeric p12 in the complex. Native Pol $\delta$ holoenzyme was immunoprecipitated by using either anti-GFP (i) or anti-p125 (ii) antibody (Fig 2A). Each of the four native subunits ( $p 125, p 68, p 50$, and p12) was detected by probing with subunit-specific antibody, whereas an anti-GFP antibody detected GFP-p12. While GFP-p12 pulled down cellular Pol $\delta$ with native p12, anti-p125 antibody precipitated Pol $\delta$ complex with both the forms of p12 (native and GFP-tagged). In both of the pull-down assays, irrespective of the antibody used, we detected the presence of five subunits of Pol $\delta$ in the beads. So, p12 indeed 
A
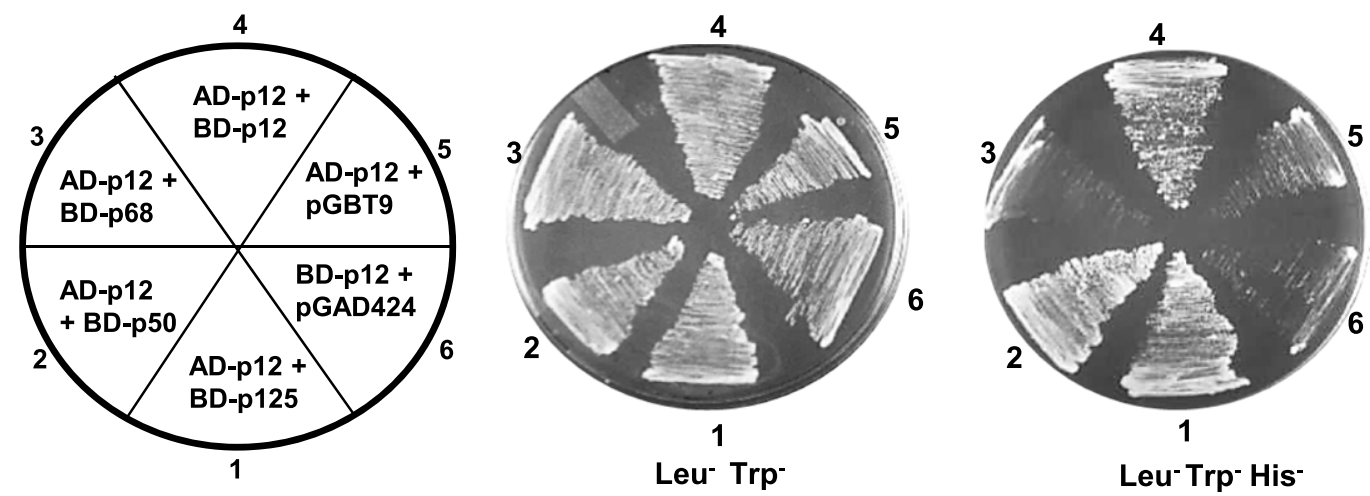

B

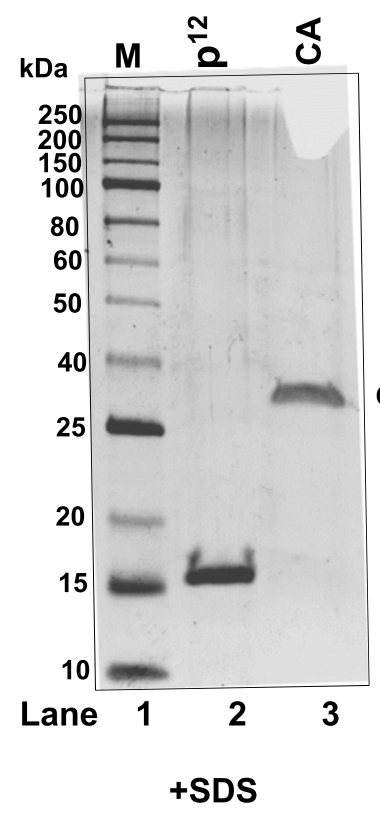

C

i.

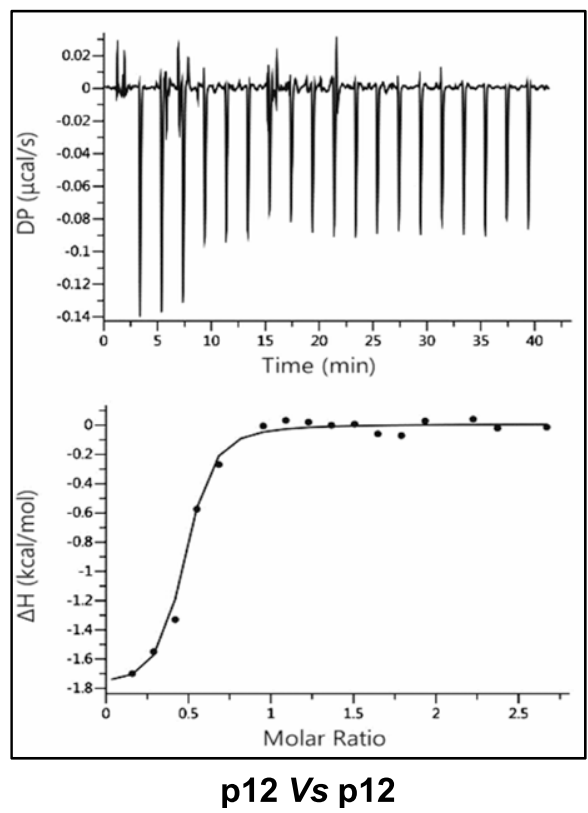

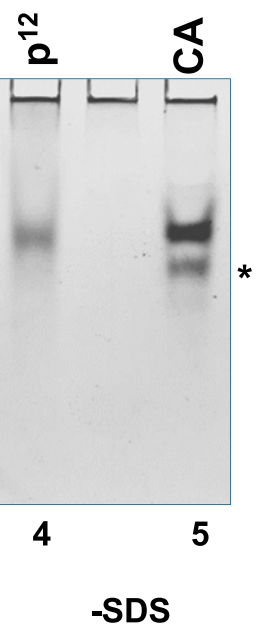

ii.

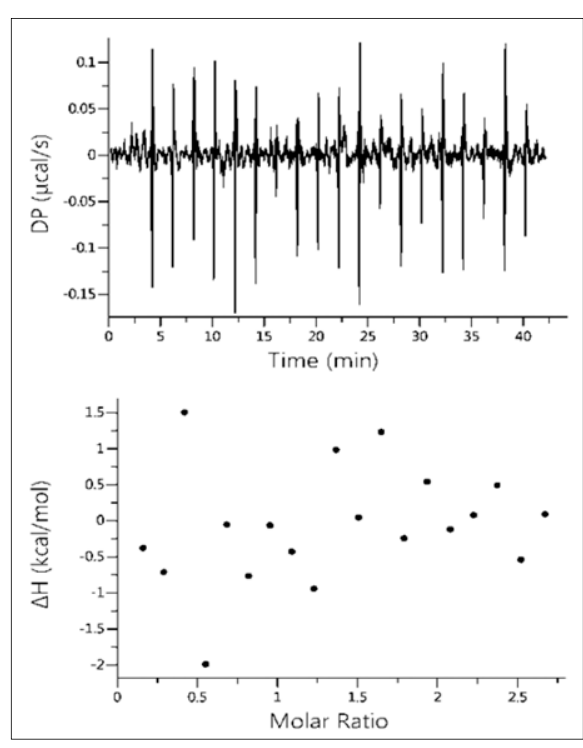

p12 Vs p12-R3A,K4A,R5A 
exists as an oligomer in the Pol $\delta$ complex even in the cellular context.

PCNA that functions as a cofactor for DNA polymerases orchestrates the replisome by recruiting multiple proteins involved in DNA transaction processes. PCNA forms distinct foci or replication factories indicative of active DNA replication entities within the nucleus (Essers et al, 2005). The p68 and p50 subunits of hPol $\delta$ were shown to form foci and co-localized with PCNA in several cell lines (Ducoux et al, 2001; Pohler et al, 2005). The CHO cells were transfected with GFP-PCNA or GFP-p12 and RFP-p12 fusion constructs to examine the physiological relevance of $\mathrm{p} 12$ oligomerization and PCNA interaction. As shown in Fig 2B, irrespective of GFP (stained green) or RFP (stained red) fusion, p12 formed discrete compact foci. The subsequent merging of foci in co-transfectant of GFP-p12 and RFP-p12 resulted in the appearance of yellow foci (i). Thus, $100 \%$ coincidental accumulation of both the p12 foci suggests that both the proteins are part of a replication unit and function together. Similarly, we have also observed subcellular co-localization of p12 with PCNA as yellow foci appeared by merging foci of GFPPCNA and RFP-p12 (ii). However, in a similar condition, we did not notice any co-localization of GFP-p12 and RFP-Pol $\theta$ foci (iii), whereas Pol $\theta$ co-localizes with PCNA (our unpublished observation). We suggest from these observations that $p 12$ could function in replication factories as a potential oligomeric protein of Pol $\delta$ with PCNA.

\section{Identification of motifs involved in p12 dimerization and interaction with PCNA}

The primary sequences of 12 from human, mouse, bovine, and $S$. pombe were aligned to identify dimerization and PIP motifs in 12 (Fig 3A). The CLUSTAL W alignment analysis showed a high degree of amino acid conservation of $\mathrm{p} 12$ sequences in mammals, and they showed only $\sim 17 \%$ identity with S. pombe orthologue Cdm1. The carboxyl termini of these proteins displayed better conservation than the amino termini. Cdm1 is composed of $160 \mathrm{aa}$, and the divergence is apparently due to possession of an insert of about 38 aa exactly in the middle of the 12 homologous sequences in Cdm1. We thought to examine the role of the two highly conserved sequences located at the extreme ends of p12: a basic tripeptide sequence ${ }_{3} R K R_{5}$ motif (henceforth referred as RKR motif) and a putative PIP box sequence ${ }_{98} \mathrm{QCSLWHLY}_{105}$ (henceforth referred as PIP motif). The putative PIP motif is located in the carboxyl-terminal tail of p12, the usual position of the PIP motif in most of the DNA polymerases, and appears to be very similar to known PIP sequences (Fig 3B). Because an earlier study reported ${ }_{4} \mathrm{KRLITDSY}_{11}$ (a part of RKR motif) as a PCNA-binding region of p12 (Li et al, 2006), we wanted to compare the model structure of this peptide with
${ }_{98} \mathrm{QCSLWHLY}_{105}$. Structurally, PIP box sequences are highly conserved, and formation of a $3_{10}$ helix is a characteristic feature of such sequences. The amino acid stretch encompassing RKR (1-MGRKRLITDSYPVK-14) and PIP (92-GDPRFQCSLWHLYPL-106) domains was used for peptide structure prediction by using PEP-FOLD3 server (http://bioserv.rpbs.univ-paris-diderot.fr/services/ PEP-FOLD3/) rather than using a known template-based prediction to avoid any bias. Furthermore, the models were validated by the SAVES and Ramachandran plot (Fig S2A and B), which showed most of the residues in allowed regions. Our structural prediction suggested that the first 10 aa of the RKR motif form an $\alpha$-helix, whereas the PIP motif of p12 forms a typical $3_{10}$ helix, the structure that fits snugly into the interdomain connecting loop (IDCL) domain of PCNA. The p12 PIP structure was further aligned with available $X$-ray crystal structures of PIP peptides from p21 (1AXC) and p68 (1U76). The superimposition shows a high degree of similarity between the PIP motifs (Fig 3C). Similarly, the p12 peptide structure was aligned with that of the p68 PIP-hPCNA co-crystal structure, and a remarkable overlapping between the structures was observed (Fig 3C). Thus, our in silico analysis indicated that the C-terminal PIP motif ${ }_{98} \mathrm{QCSLWHLY}_{105}$ is the most probable motif that interacts with PCNA other than the $\mathrm{N}$-terminal RKR motif ${ }_{4}$ KRLITDSY $_{11}$.

Two p12 mutants were generated by mutating residues R3, K4, R5 and L104, Y105 to alanines; and their interaction with wild-type p12 and PCNA was analyzed by yeast two-hybrid approach for providing experimental evidence to our in silico prediction (Fig 3D). As depicted, whereas transformants of BD-p12 with AD-PCNA or AD-p12 grew on SDA plate lacking leucine, tryptophan, and histidine amino acids (rows 5 and 6), both R3A, K4A, R5A and L104A, Y105A p12 mutants were unsuccessful in interacting with PCNA, and thus, no growth was observed (rows 2 and 4), including the vector control (rows 7 and 12). Interestingly, R3A, K4A, R5A mutant is also defective in p12 interaction in yeast cells but not the L104A, Y105A mutant (compare row 1 with row 3 ). These in vivo results suggest that whereas the RKR motif plays a dual role in dimerization and PCNA interaction, ${ }_{98} \mathrm{QCSLWHLY}_{105}$ is involved only in PCNA binding.

Furthermore, we wanted to examine whether dimerization of p12 is also required for other Pol $\delta$ subunit interactions. Yeast twohybrid analyses of co-transformants harboring AD-R3A, K4A, R5A with BD-p125 or BD-p50 demonstrated that mutation in this motif does not affect Pol $\delta$ subunit interaction, as transformants grew efficiently on the Leu- Trp- His- plate (Fig 3D, sectors 8-11). Thus, dimerization of $\mathrm{p} 12$ is not required for p125 or p50 binding. However, it is not clear whether p125 and p50 bind to the same or different regions in p12. Nonetheless, dimerization could facilitate binding of p125 and p50 subunits of Pol $\delta$ to separate monomer of the p12 dimer.

\section{Figure 1. Interaction of p12 with hPol $\delta$ subunits.}

(A) Yeast two-hybrid analysis showing the interaction of p12 with the various subunits of hPolס. HFY7C yeast transformants with various GAL4-AD and GAL4-BD fusions were selected on SD media plates lacking leucine and tryptophan, and with and without histidine amino acid. Sector 1, AD-p12 + BD-p125; Sector 2, AD-p12 + BD-p50; Sector 3, AD-p12 + BD-p68; Sector 4, AD-p12 + BD-p12; Sector 5, AD-p12 + pGBT9; and Sector 6, AD-p12 + pGAD424. (B) The purified p12 protein was resolved in native and SDS-PAGE gels. Lane 1: MW; lanes 2 and 4: p12; and lanes 3 and 5: CA. * indicates degraded CA protein. (C) ITC analysis of p12 to wild-type (i) or RKR-mutant p12 (ii). In each panel, the upper half shows the measured heat exchanges during each protein injection. The lower half of each panel shows the enthalpic changes as a function of the molar ratio of p12 to wild-type or RKR-mutant p12 monomer. Circles and lines denote the raw measurements and the fitting to a one set of identical sites. Source data are available for this figure. 
A

\section{i. IP using anti-GFP-p12 ii. IP using anti -p125}

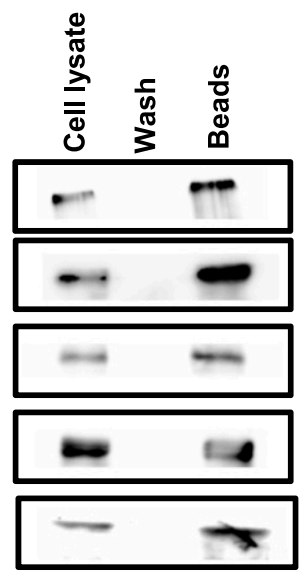

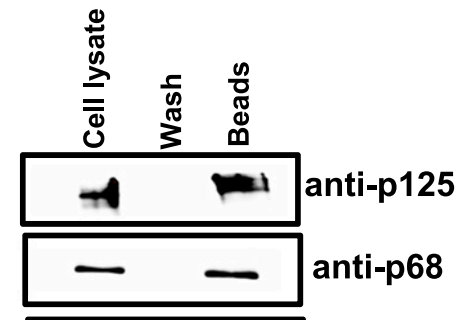
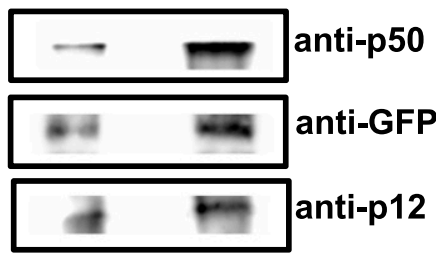

anti-GFP for GFP fused $p 12$ anti-p12

\section{B}

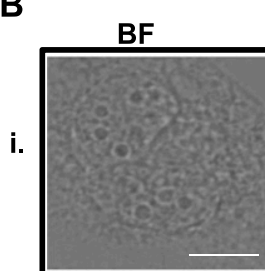

GFP-p12

RFP-p12
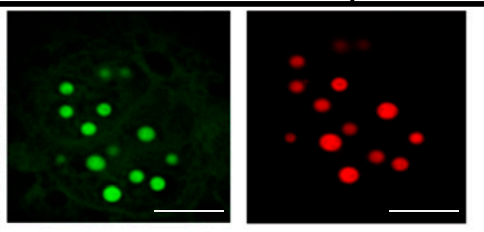

GFP-PCNA

RFP-p12

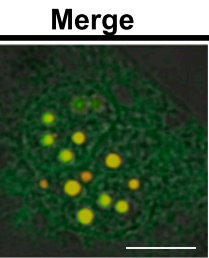

$\mathrm{BF}$
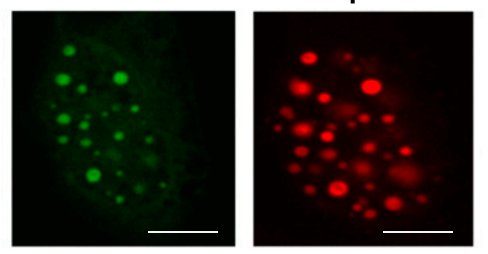

Merge

ii.

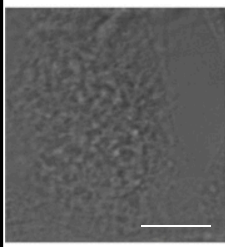

BF

\section{GFP-p12}

RFP-Pol $\theta$

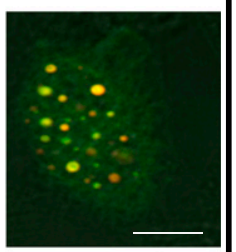

iii.
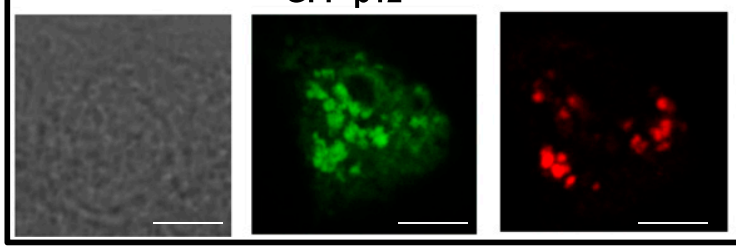

Merge

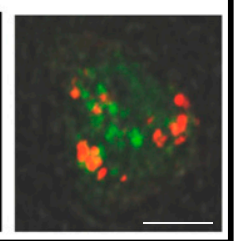

\section{RKR motif of p12 is critical for dimerization}

Multiple basic amino acid motifs such as RKR/KKR/KRK in other proteins are known to play crucial roles in a variety of cellular processes such as their retention and exit from ER, and nuclear localization, as well as the gating of $\mathrm{K}^{+}$channels (Joiner et al, 1997; Zerangue et al, 1999; Fagerlund et al, 2002; Jones et al, 2007). Such motifs are also found to be involved in protein-protein interaction (van Hennik et al, 2003; Wang et al, 2012; Collins et al, 2014). The mutant proteins R3A, K4A, R5A and L104A, Y105A were purified to near homogeneity and analyzed in SDS-PAGE to find out the involvement of the RKR motif in p12-p12 dimerization (Fig S3, lanes 3 and 4). The wild-type and mutant p12 proteins resolved at a similar position in denaturing PAGE. Thus, mutations in these residues had
Figure 2. Existence of p12 oligomers in the cellular context of Pold.

(A) Native hPol $\delta$ was co-immunoprecipitated from HEK293 cells transfected with GFP-p12. Either anti-GFP (i) or anti-p125 (ii) antibody was used to

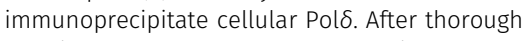
washings, the eluate was separated in $12 \%$ SDS-PAGE, and the presence of various subunits of hPol $\delta$ was detected by the subunit-specific antibody. GFP-p12 was detected by the anti-GFP antibody. (B) Nuclear colocalization of $\mathrm{p} 12$ and PCNA. CHO cells were COtransfected with GFP-p12 and RFP-p12 (i), GFP-PCNA and RFP-p12 (ii), or GFP-p12 and RFP-Pol $\theta$ (iii). After 48 h, the cells were fixed and mounted as described in the

Materials and Methods section, and images were taken using Leica TCS SP5 at $63 \times$ objective. Scale bar is equal to $5 \mu \mathrm{m}$.

Source data are available for this figure. 
A

M. musculus

B. taurus

H. sapiens

S. pombe
Dimerization motif

p12 (1-32 aa) MGRKRF - - - - - ITDSYPVVKKREGP PGHSKGELAPEL- - - - - -

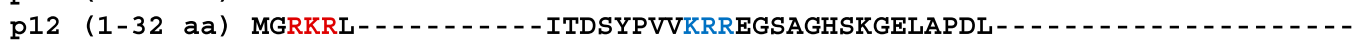

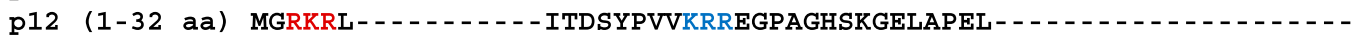

Cdm1 (1-60 aa) M-KKRTTQAKKSGQNTNIRDVFPHVVRSNSSQSHIGKKVSSEQSPTPDVTITTKTLDERIKEDD *:*. * * : : . * : : : :
M. musculus

B. taurus

H. sapiens

S. pombe p12 (33-72 aa) --- (33-7 aa)

p12 (33-72 aa) - - - - - -

p12 (33-72 aa) ------------GEEPQPRDEEEAELELLRQFDLAWQYGPCTGITRLQRWCRAKQ

Cdm1 (61-120 aa) ELSKEVEEAWNQIMAERISEPIHCENITKVEFILHHFDTTARYGPYLGMTRMQRWKRAKN

PIP

M. musculus p12 (73-107 aa) MGLKPPLEVYQVLKAHPEDPHFQCSLWHLYPL----- 107

B. taurus p12 (73-107 aa) MGLKPPPEVHQVLQSHPGDPRFQCSLWHFYPL----- 107

H. sapiens p12 (73-107 aa) MGLEPPPEVWQVLKTHPGDPRFQCSLWHLYPL----107

$S$. pombe Cdm1 (121-160 aa) FNLNPPETVGKILMLEEADEENRKRESLFYDLQTIPG 160

B

ScPol $\delta$ - Pol3 995 KGGLMSFI 1003

ScPol $\delta$ - Pol31 321 DKSLESYF 328

ScPol $\delta$ - Pol32 331 QGTLESFF 347

hPol $\delta$ - p68 456 QVSITGFF 463

hPol $\delta$ - p50 $57 \quad$ LIQMRPFL 64

hPOI - P12 $98 \quad$ QCSLWHLY 105

hPol $\delta$ - p12 4 KRLITDSY 11

ScRad30-pip 618 SKNILSFF 625

hRad30 pip2 437 STDITSFL 444

hRad30 pip1 701 MQTLESFF 708

Consensus QXXLXXFF
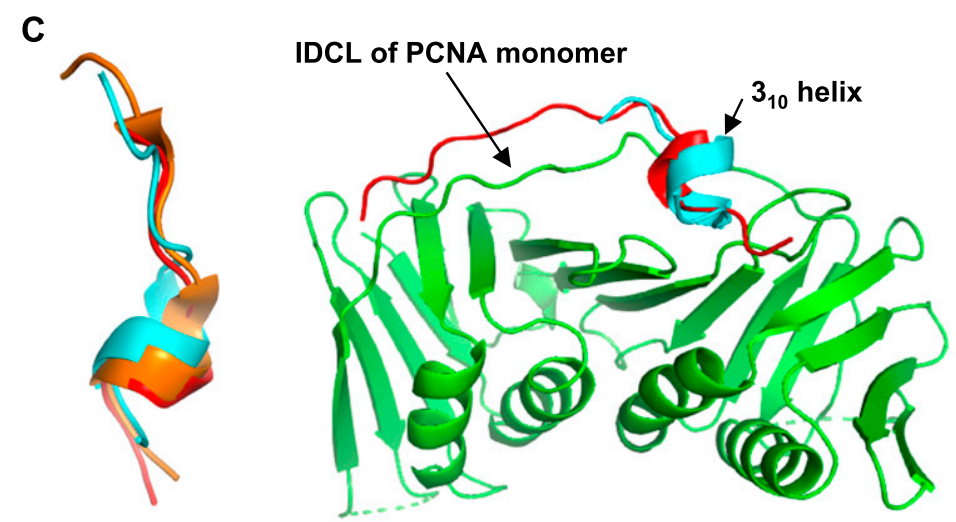

$3_{10}$ helix

D

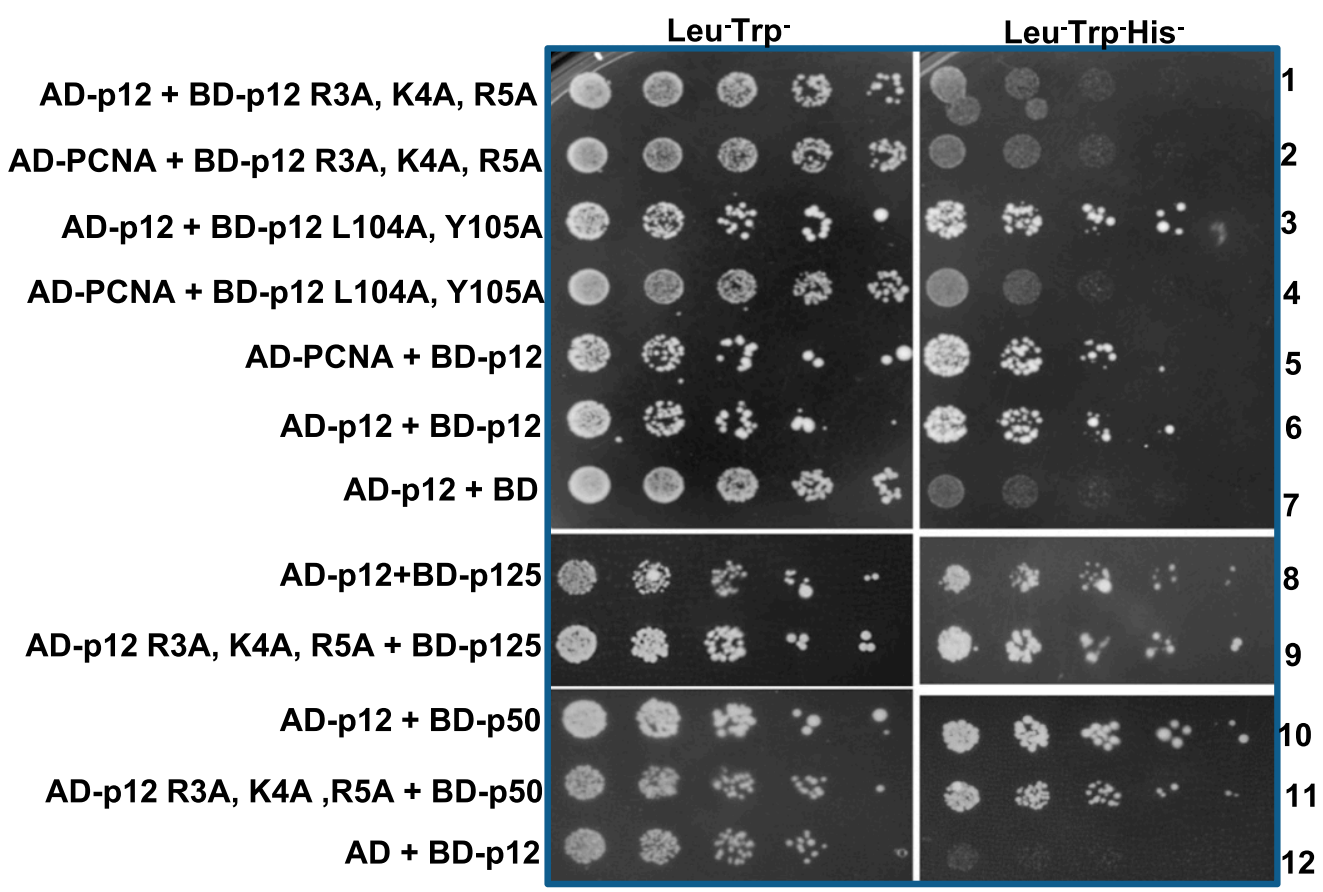




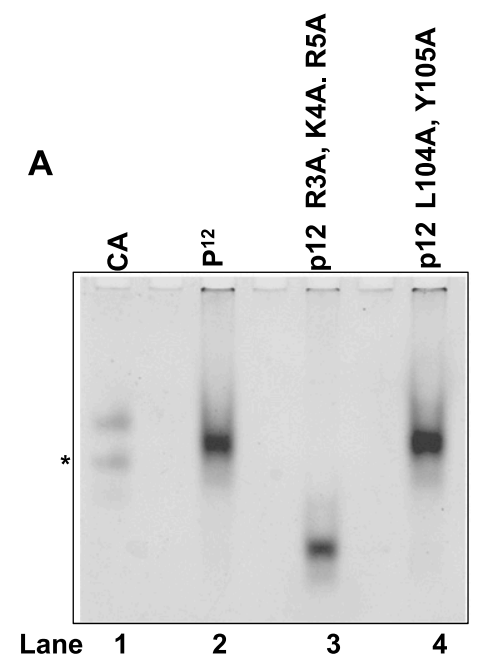

B

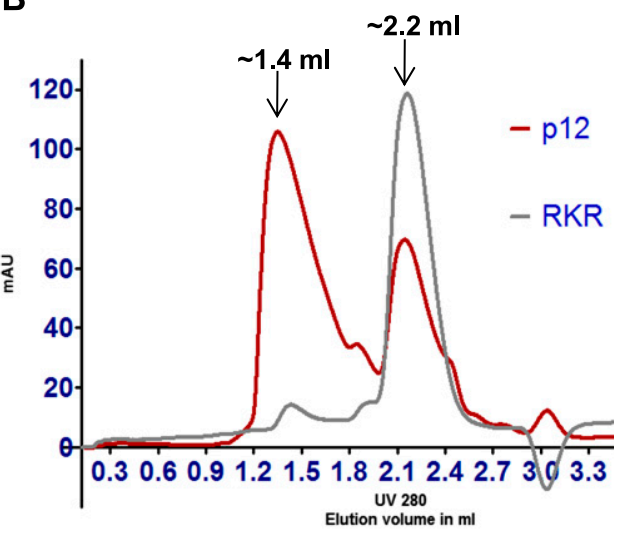

Figure 4. RKR motif is involved in dimerization. (A) R3A, K4A, R5A and L104A, Y105A p12 proteins were resolved in native PAGE. Lane 1: CA; lane 2: p12; lane 3: R3A, K4A, R5A; and lane 4: L104A, Y105A. * represents degraded CA. (B) About $10 \mu \mathrm{g}$ of wild-type (red line) and R3A, K4A, R5A (grey line) p12 proteins were subjected to size-exclusion chromatography. Two elution peaks at $\sim 1.4$ and $\sim 2.2 \mathrm{ml}$ were observed representing dimer and monomer populations, respectively. (C)

Immunoprecipitation of GFP-p12 by FLAG-p12 (lane 3) but not of GFP-R3A, K4A, R5A mutant (lane 5). Lane 1: cell lysate input; lanes 2 and 4: washings from beads. Source data are available for this figure.

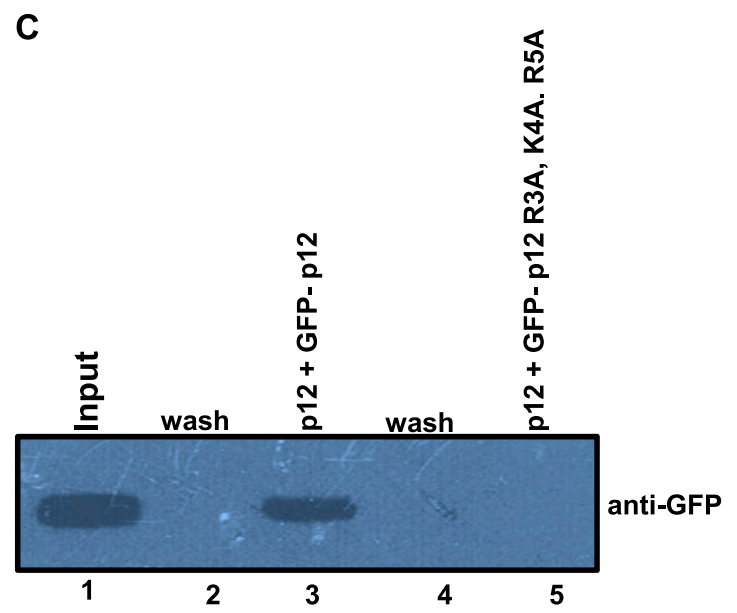

wild-type p12 protein eluted in two peaks of volume at $\sim 1.4$ and $\sim 2.2 \mathrm{ml}$, corresponding to an oligomeric and monomeric state of the protein (red line), R3A, K4A, R5A mutant eluted (grey line) as a single peak at a volume of $2.2 \mathrm{ml}$ (Fig 4B). This demonstrates the dimerization of $\mathrm{p} 12$ mediated by the RKR motif. This result also rules out any change in stokes radius and residual charge of p12 protein causing abnormal migration in native PAGE due to mutations in the dimerization motif. Co-immunoprecipitation experiment was also carried out in the cell lysates harboring
FLAG-p12 and GFP-p12 or GFP-p12 R3A, K4A, R5A by using anti-FLAG antibody-conjugated beads; and further probed with an anti-GFP antibody (Fig 4C). Although anti-FLAG antibody could pull down wild-type p12 as detected by the anti-GFP antibody, it did not precipitate the RKR mutant (compare lanes 3 and 5). Our yeast two-hybrid, native PAGE, size exclusion chromatography, and pulldown assays demonstrated that indeed RKR motif is a proteinprotein interaction motif, and in this study, we show that it is essential for p12 dimerization.

Figure 3. Identification of p12 motifs involved in dimerization and PCNA interaction.

(A) The primary sequences of the fourth subunit of Polઠs from human (Homo sapiens), mouse (Mus musculus), bovine (Bos taurus), and fission yeast (S. pombe) were aligned by CLUSTALW. Conserved dimerization motif and PIP-box sequences are colored brown. Identical residues are marked with *, conserved residues are marked with :, and less conserved residues are marked with $\cdot$ (B) Identified PIP motif residues from DNA polymerase $\eta$ and $\delta$ subunits were aligned and compared with putative PIP sequences in p12. Repeated residues in more than one sequences are highlighted. (C) Superimposition of model $3_{10}$ helix structure of p12 PIP (cyan) with available PIP structures from p21 (orange) and p68 (red) peptides without and with human PCNA monomer. (D) Yeast two-hybrid analysis showing the interaction of mutants p12 with wild-type p12 and PCNA. HFY7C yeast transformants with various GAL4-AD and GAL4-BD fusions were selected on SD media plates lacking leucine and tryptophan, and with and without histidine amino acids. Row 1: AD-p12 + BD-p12 R3A, K4A, R5A; row 2: AD-PCNA + BD-p12 R3A, K4A, R5A; row 3: AD-p12 + BD-p12 L104A, Y105A; row 4: AD-PCNA + BD-p12 L104A, Y105A; row 5: AD-PCNA + BD-p12; row 6: AD-p12 + BD-p12; row 7: AD-p12 + pGBT9; row 8: AD-p12 + BD-p125; row 9: AD-p12 R3A, K4A, R5A + BD-p125; row 10: AD-p12 + BD-p50; row 11: AD-p12 R3A, K4A, R5A + BD-p50; and row 12: AD-p12 + pGBT9.

Source data are available for this figure. 


\section{Formaldehyde cross-linking reveals dimerization of p12}

Our native PAGE and ITC analyses suggested potential dimeric nature of 12 as it was migrating at a similar position with CA (30 kD). To estimate the exact number of p12 molecules in the oligomeric complex, we used a formaldehyde cross-linking assay. Reagents such as formaldehyde or glutaraldehyde cross-links neighboring lysine or arginine residues of proteins to form a stable complex that can even withstand SDS denaturation (Manohar \& Acharya, 2015); moreover, our mutational analysis deciphered the involvement of the RKR motif in dimerization. Therefore, purified recombinant proteins were cross-linked with formaldehyde, analyzed on $12 \%$ SDS-PAGE, and detected by Coomassie brilliant blue staining (Fig 5A). Upon treatment with the cross-linker, both wild-type and L104A, Y105A mutant p12 proteins showed concentration-dependent cross-linked dimers (lanes 2, 3, 4, 10, 11, and 12) that were migrating below $32 \mathrm{kD}$ position and did not form any higher order oligomers, whereas R3A, K4A, R5A mutant protein remained as monomer (lanes 6, 7, and 8). Without any cross-linker, all the three proteins migrated to the bottom of the gel (lanes 5, 9, and 13). In addition to the ${ }_{3} \mathrm{RKR}_{5}$ motif, mammalian p12 possesses yet another multibasic motif ${ }_{15} \mathrm{KKR}_{17}$ (Fig 3A, colored in blue). As R3A, K4A, R5A p12 mutant failed to form any oligomer in the presence of formaldehyde, it also implies that ${ }_{15} \mathrm{KKR}_{17}$ sequence has no role in dimerization, and the dimeric property of $\mathrm{p} 12$ is specifically attributed by the $R_{3} K_{4} R_{5}$ motif. Even the ITC assay failed to detect any binding between wild-type p12 and R3A, K4A, R5A p12 mutant (Fig 1C, ii).

Because we did not detect dimerization of the RKR motif mutant in any of our assays, we wanted to rule out the possibility that this effect resulted from a significant change in p12 conformation. For this reason, we compared the circular dichroism (CD) spectra of the wild-type and R3A, K4A, R5A mutant p12 proteins (Fig 5B). The CD spectra determined in the "far-UV" region (200 to $260 \mathrm{nM}$ ) showed p12 to be enriched in $\alpha$-structure as evident from the characteristic negative peaks at $208 \mathrm{~nm}$ and $222 \mathrm{~nm}$. It also indicates that the mutant protein retains a similar level of secondary structures as that of the wild-type protein, which would suggest that the mutations do not cause a major perturbation to the p12 structure.

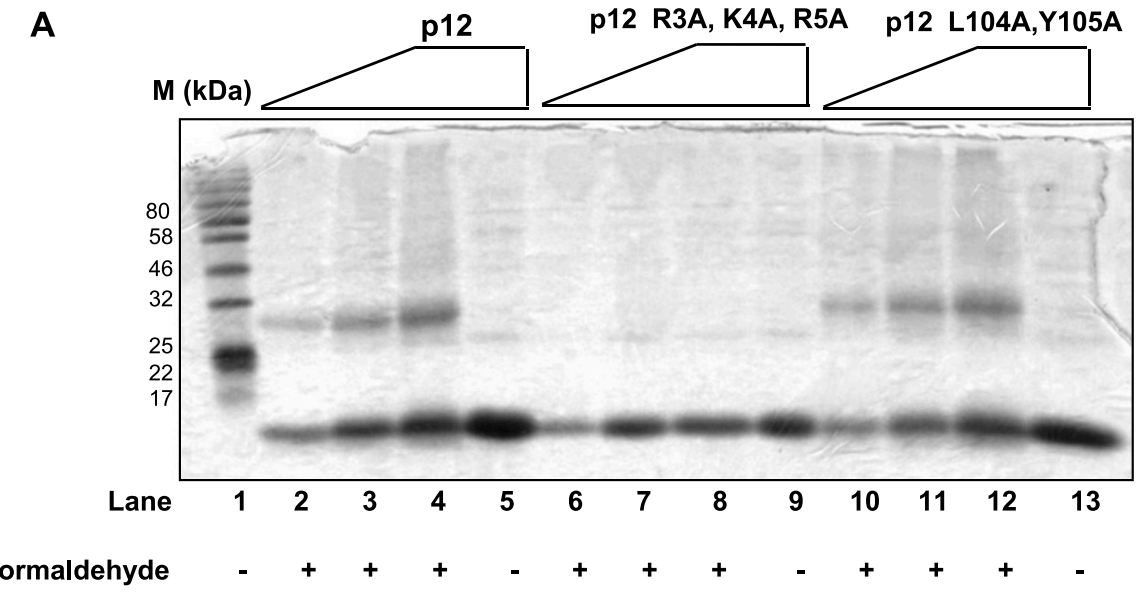

B

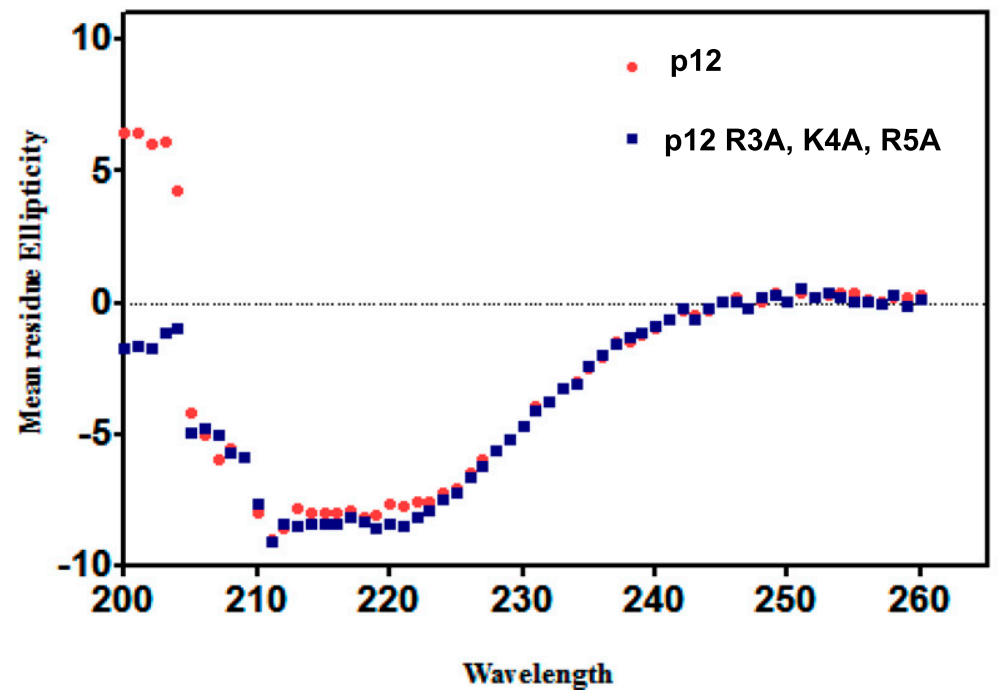

Figure 5. Formaldehyde cross-linking of $\mathrm{p} 12$ proteins. (A) About 1-5 $\mu \mathrm{g}$ of p12 proteins were cross-linked with $0.5 \%$ formaldehyde solution for $30 \mathrm{~min}$ at $25^{\circ} \mathrm{C}$. After termination with SDS sample buffer, they were resolved in a 12\% SDS-PAGE. Lane 1: MW; lanes 2-5: p12; lanes 6-9: R3A, K4A, R5A; and lanes 10-13: L104A, Y105A. Lanes 5, 9, and 13: proteins treated similarly but without

formaldehyde. (B) Far UV-CD spectra of wild-type (red) and R3A, K4A, R5A mutant (blue) p12. CD spectra at pH 7.5 between 200 and $260 \mathrm{~nm}$ were recorded. Data represent values determined after solvent correction and after averaging each set $(n=3)$.

Source data are available for this figure. 
Considering all these pieces of evidence, we conclude that p12 forms a dimer solely mediated by the $R_{3} K_{4} R_{5}$ motif.

\section{In vitro reconstitution of pentameric human Pol $\delta$ holoenzyme}

Various subassemblies of hPol $\delta$ such as p125 alone, p125-p50, p125p50-p68, and p125-p50-p68-p12 have been purified in vitro by mixing various combinations of purified proteins (Xie et al, 2002; Zhou et al, 2012). In this study, Pol $\delta$ holoenzyme was expressed by co-transforming two bacterial expression constructs GST-p125 and pCOLA234 (p50-p68-His-FLAG-p12), and the complex was purified using glutathione-sepharose beads to near homogeneity. Taking advantage of the strategically located PreScission protease site, the

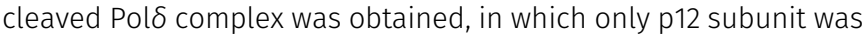
amino-terminally FLAG-tagged. We refer this complex as the first Pol $\delta$ complex. To conclusively show the two different forms of 12 in the holoenzyme, untagged 12 protein purified using bacterial GST- p12 system was mixed to the first Pol $\delta$ complex and the mixture was incubated at $4^{\circ} \mathrm{C}$ for $4 \mathrm{~h}$. If p12 forms a dimer in the Pol $\delta$ complex, untagged p12 will compete out some of the resident FLAG p12 and a reorganized Pol $\delta$ with five subunits will appear. Thus, the mixture was loaded into $S_{200}$ mini-column for separation and the fractions were collected in a 96-well plate. As we could not detect enough protein by Coomassie staining despite our repeated trials, various fractions were analyzed in SDS-PAGE followed by detection of various subunits by probing with a specific antibody (Fig 6). The membrane was probed with an anti-p68 antibody (A) and selected fractions again resolved in SDS-PAGE (B) to detect the presence of enriched holoenzyme fractions. As depicted in the figure, initial fractions were enriched in Pol $\delta$ holoenzyme (A8-B2) and a clear shift of untagged p12 proteins towards the complex was also noticed. Interestingly, early fractions such as A8-A10 showed a majority of FLAG-p12 and very little amount of untagged p12 (first complex), whereas the later fractions convincingly showed the
A

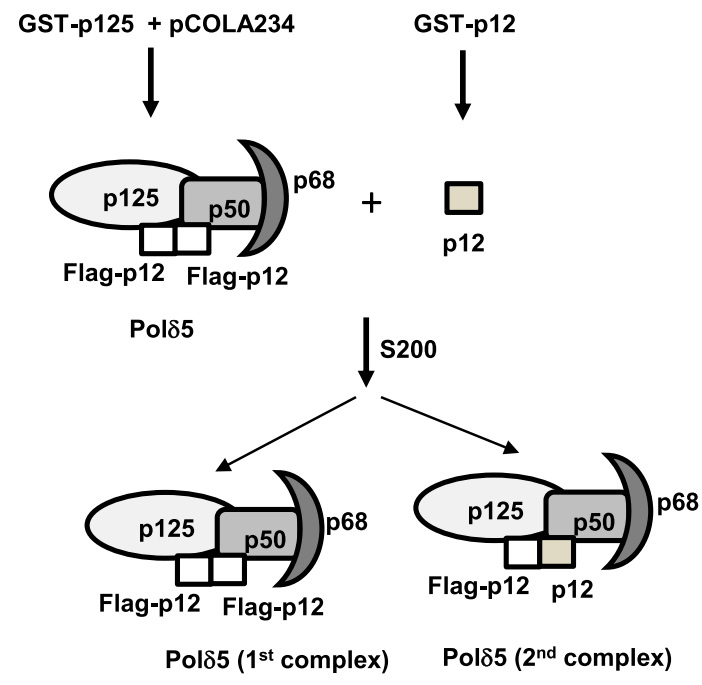

Figure 6. Purification of the pentameric hPolø holoenzyme.

Schematic representation of purification of pentameric Pol $\delta$ was shown. A mixture of Pol $\delta 4$ and p12 was separated by gel filtration chromatography, and various fractions were first analyzed by probing with anti-p68 antibody. (A) Enriched fractions were again separated in SDS-PAGE and further transferred to PVDF membrane. The membrane was cut into pieces as per the molecular weight of various subunits and was individually probed with a specific antibody. (B) The fractions possessing Pol $\delta 5$ are denoted. Name of the fractions is as per the collections in the 96-well plate.

Source data are available for this figure.

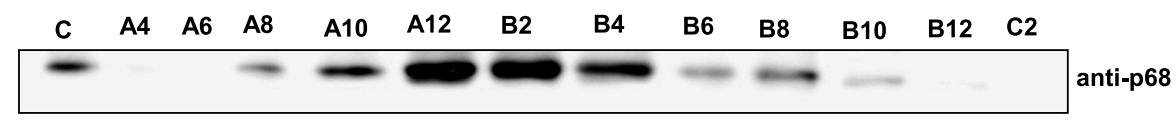

B

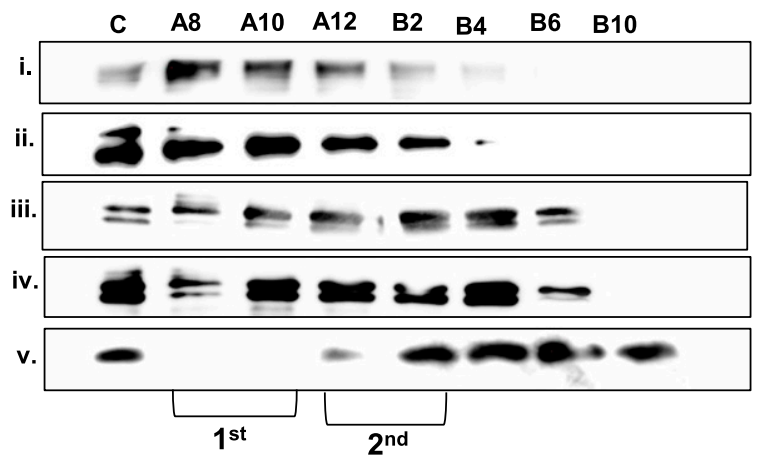

Pentameric Pol反 anti p125

anti p68

anti p50

anti p12 (FLAG-p12)

anti p12 (p12) 
presence of both tagged and untagged p12 subunits (A12-B2, second complex). The faster elution of the first complex from the size-exclusion column suggests that the early eluate is also a pentameric complex. In this approach, we could purify two

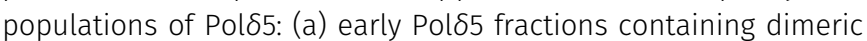
FLAG-p12 (A8-A10) and (b) later Pol $\delta 5$ fractions where some of the FLAG-p12 were replaced with untagged p12 (A12-B2). This analysis demonstrates that Pol $\delta 5$ is the predominant form and it is unlikely

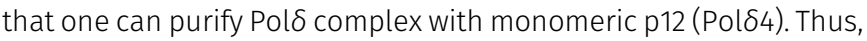
in our opinion earlier reported, purified and functionally characterized Pol 84 holoenzyme could very well be the Pol $\delta 5$ complex (11, $15,23,24$, and 39 ). Considering both our in vivo and in vitro analyses, we propose that $h$ Pol $\delta$ is intrinsically a pentameric complex, possessing two p12 subunits.

\section{Dimerization of $\mathrm{p} 12$ is essential for PCNA interaction}

Because both $\mathrm{R}_{3} \mathrm{~K}_{4} \mathrm{R}_{5}$ and ${ }_{98} \mathrm{QCSLWHLY}{ }_{105}$ motifs are required for PCNA interaction and the former motif additionally is involved in protein dimerization, to ascertain any regulatory role of this motif in p12 function, a PCNA overlay experiment was carried out. Proteins were resolved on native PAGE to keep the natural folding and the dimer structure intact (as carried out in Fig 4A), transferred to PVDF membrane and blocking was performed in the presence of PCNA. After several washings, the blot was developed with the anti-PCNA antibody (Fig 7A). As depicted in the figure, although p12 and its L104A, Y105A mutant formed dimers (upper panel, compare lanes 1 and 3), a signal for only wild type was detected (lower panel), suggesting that the ${ }_{98} \mathrm{QCSLWHLY}_{105}$ motif is the true PIP motif required for PCNA interaction. Despite retaining the PIP motif in the extreme $C$-terminal tail, because of its inability to form a dimer, the R3A, K4A, R5A mutant failed to bind to PCNA. Similarly, a pull-down experiment was also carried out by taking a mixture of stoichiometry equivalents of purified GST-p12 or various p12 mutants and PCNA in Tris-buffer containing $150 \mathrm{mM} \mathrm{NaCl}$ salt concentration (Fig 7B). The mixture was incubated with GST beads at $4^{\circ} \mathrm{C}$ for $3 \mathrm{~h}$ in a rocking condition; beads were washed thrice and the bound PCNA was eluted by SDS-containing sample buffer. The eluted PCNA could be detected by anti-PCNA antibody only when it was mixed with wildtype p12 but not with the L104A, Y105A or R3A, K4A, R5A mutant (compare lane 3 with lanes 6 and 9).

ITC assay was carried out by placing p12 $(10 \mu \mathrm{M})$ in the sample cell and titrated by PCNA $(120 \mu \mathrm{M})$ injection to determine the binding affinity of p12 with PCNA. We did not observe any significant change in heat when p12 or PCNA was injected to the cell containing a buffer (Fig S4). Upon p12-PCNA binding, the heat was liberated and kinetic parameters such as $\Delta H, \Delta G$, and the $K_{D}$ for the complex were recorded as $-80 \mathrm{kcal} / \mathrm{mol},-6.80 \mathrm{kcal} / \mathrm{mol}$, and $10 \mu \mathrm{M}$, respectively (Fig $7 \mathrm{C}$ ). In a similar assay condition, no significant heat exchange was observed in a titration where RKR- or PIP-motif p12 mutants were kept in the cell and PCNA in the syringe, suggesting no detectable interaction between the proteins. Thus, our results suggest that dimerization at the $\mathrm{R}_{3} \mathrm{~K}_{4} \mathrm{R}_{5}$ motif promotes p12 interaction with PCNA via the ${ }_{98} \mathrm{QCSLWHLY}_{105}$ motif.

\section{Interdomain connecting loop region of hPCNA mediates its interaction with $\mathrm{p} 12$}

As our predicted model structure showed p12 PIP peptide binding to IDCL domain of hPCNA, yeast two-hybrid assay was carried out with p12 fused to Gal4-binding domain and two PCNA mutants, namely, pcna-79 and pcna-90, fused to Gal4 activation domain. In pcna-79, two key hydrophobic residues L126 and 1128 of the interdomain connecting loop were mutated to alanines, whereas pcna-90 possesses the two mutations P253A and K254A in the extreme C-terminal tail of PCNA. Most of the interacting proteins bind to any of these two regions of a trimeric PCNA ring (Manohar \& Acharya, 2015). While wild type and pcna-90 were able to interact with p12 as evident from the growth on the SDA plate lacking leucine, uracil, and histidine (Fig 8A, sectors 1 and 3), pcna-79 did not support the survival as it failed to form intact Gal4 by interacting with p12 (sector 2). The p12 PIP mutant was used as a negative control (sector 4). To strengthen our finding, GST pull-down assay was carried out. An equal stoichiometry of wild type and IDCL mutant of PCNA proteins was incubated with GST-p12 and pull-down assays were performed on glutathione-sepharose affinity beads as described previously (Acharya et al, 2005). As can be seen from the data shown in Fig 8B, although GST-p12 was able to pull down most of the wild-type PCNA from solution (compare lane 1 with lane 3), it failed to bind pcna-79 protein (compare lane 4 with lane 6 ) as detected by the anti-PCNA antibody.

\section{Cdm1, a p12 homologue of S. pombe also forms a dimer}

The other fourth subunit of DNA polymerase $\delta$ that has been well characterized is Cdm1, a p12 orthologue from S. pombe (Zuo et al, 2000). Cdm1 consists of $160 \mathrm{aa}$, has a molecular mass of $18.6 \mathrm{kD}$, and a pl of 7.73. Like p12, it shows abnormal mobility in the SDS-PAGE and migrates as $\sim 22-\mathrm{kD}$ protein (Fig 9A). As it also has a conserved RKR motif $\left(\mathrm{K}_{2} \mathrm{~K}_{3} \mathrm{R}_{4}\right.$ in $\left.\mathrm{Cdm} 1\right)$ at its $\mathrm{N}$-terminal end, we wanted to examine whether the homodimerization property of the smallest subunits of Pol $\delta$ is evolutionarily conserved. Wild-type and K2A, K3A, R4A mutant Cdm1 proteins were purified to near homogeneity from bacterial overexpression system and analyzed on native PAGE. Just like p12, the wild-type Cdm1 migrated slower than its mutant. The slower migration of Cdm1 complex in comparison with p12 dimer could be attributed to the differences in their pl and MW ( $\mathrm{pl}$ 7.3 versus 6.3; MW 12 versus 18.6). The dimeric p12 incidentally migrates at about the same position with monomeric Cdm1-mutant protein (Fig 9B). Thus, we concluded that homodimerization is the intrinsic property of the fourth subunit of Pol $\delta$ and is mediated by conserved basic amino acids located at the extreme amino terminal end (RKR/KKR motif).

\section{Discussion}

DNA polymerase $\delta$ is a high-fidelity essential DNA polymerase. It not only plays a central role in DNA replication but also participates in DNA recombination and several DNA repair pathways from yeasts to human (Hindges \& Hubscher, 1997; Burgers, 1998). 
A

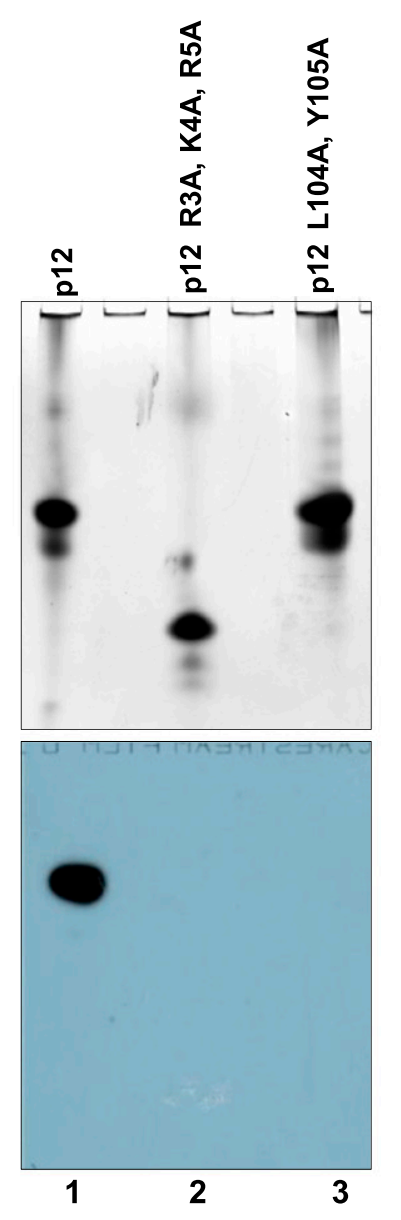

C
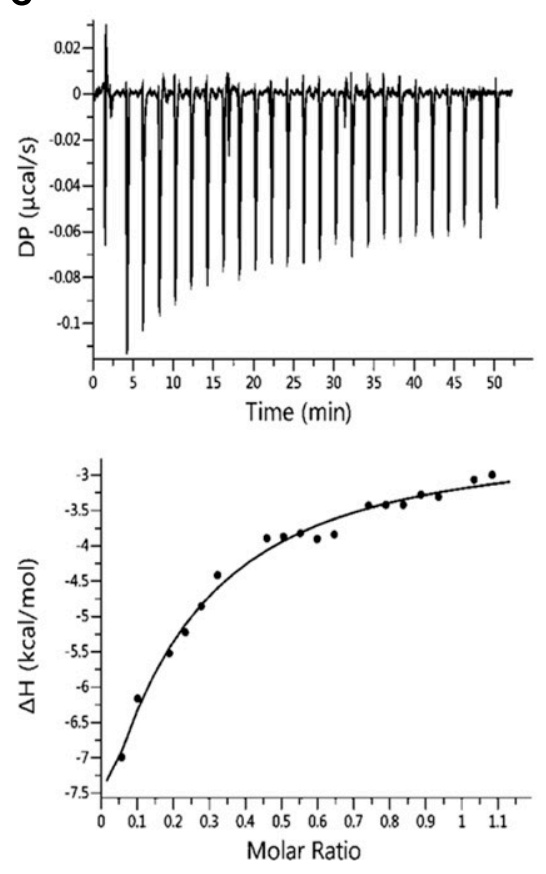

i. p12 and PCNA
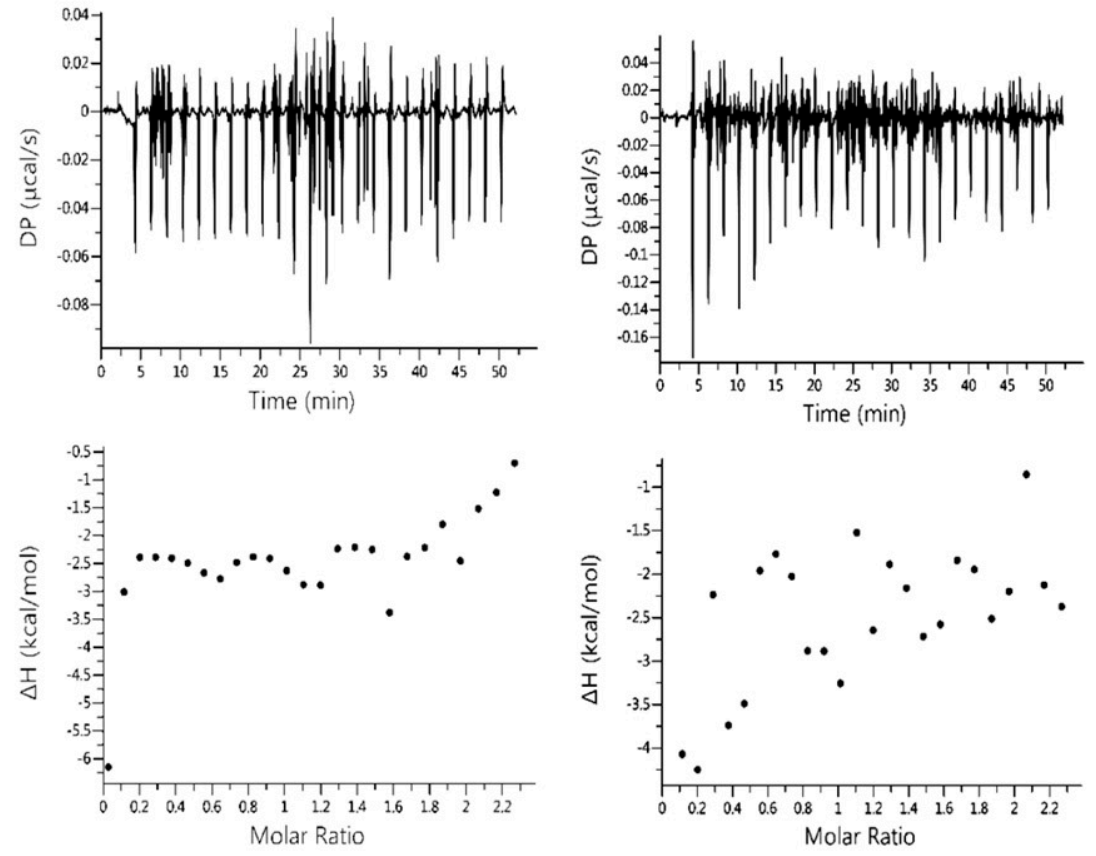

ii. p12 R3A, K4A, R4A and PCNA

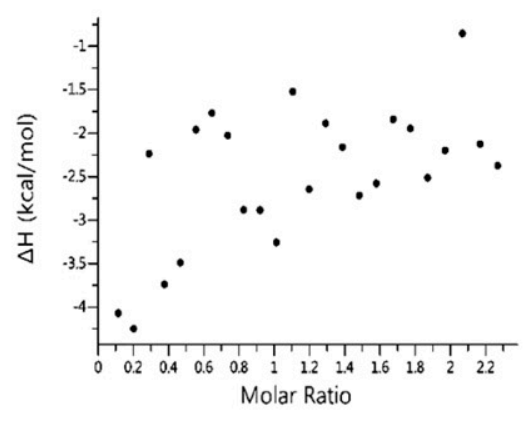

iii. p12 L104A, Y105A and PCNA 


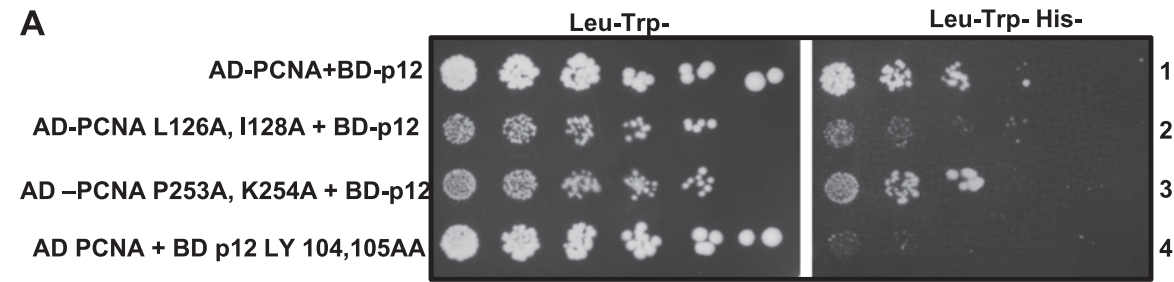

B

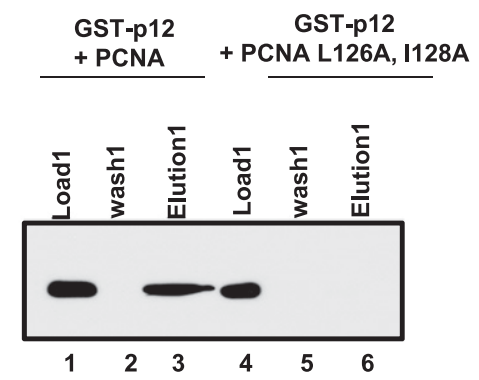

Figure 8. IDCL of hPCNA binding to p12.

(A) Yeast two-hybrid analysis showing the interaction of PCNA with p12. HFY7C yeast transformants with various GAL4-AD and GAL4-BD fusions were selected on SD media plates lacking leucine and tryptophan, and with and without histidine amino acids. Row 1: AD-PCNA + BDp12; row 2: AD-PCNA L126A, I128A + BD-p12; row 3: ADPCNA P253A, L254A + BD-p12; and row 4: AD-PCNA + BDp12 L104A, Y105A. (B) GST pull down of PCNA by wild-type p12. Beads of GST-p12 were mixed with wild-type (lanes 1-3) or L126A, I128A mutant PCNA (lanes 4-6) in equilibration buffer after the incubation beads were washed, and the bound PCNA was eluted by the protein loading dye. Various fractions were resolved in $12 \%$ SDS-PAGE, blotted to the membrane, and developed by the anti-PCNA antibody. Lanes 1 and $4: 10 \%$ of load; lanes 2 and 5: $10 \%$ of third washings; and lanes 3 and 6 : total elutes.

Source data are available for this figure.
Several mutations in mouse and hPol $\delta$ subunits have been mapped to cause various cancers (Flohr et al, 1999; Goldsby et al, 2002; Albertson et al, 2009). Thus, it is important to understand the function of each subunit and their precise role in processivity and fidelity of the holoenzyme. In this report, we have reinvestigated the role of the smallest subunit of hPol $\delta, \mathrm{p} 12$, in the holoenzyme architecture and PCNA interaction.

As reported earlier and also from this study, we understand that p12 subunits interact with p125 and p50, whereas p50 makes a connecting bridge between p125 and p68 subunits (Lee et al, 2017). Thus, hPol $\delta$ is widely considered to be a heterotetrameric holoenzyme. Our yeast two-hybrid assays, cellular co-localization assay in replication foci, and co-immunoprecipitation assays suggest an oligomerization status of the p12 subunit. Using several physiobiochemical and mutational analyses of p12 proteins, further, we extrapolate our in vivo analyses to suggest that p12 exists as a homodimer in vitro mediated by the ${ }_{3} \mathrm{RKR}_{5}$ motif, which argues against the tetrameric nature of $h P o l \delta$. As it has been previously shown, various subassemblies of $h$ Pol $\delta$ holoenzymes could exist in the cell based on Pol $\delta$ 's function in either replication or repair (Zhang et al, 2016). We propose that among these sub-complexes, pentameric Pol $\delta$ is the native form of Pol$\delta$. Pull down of cellular

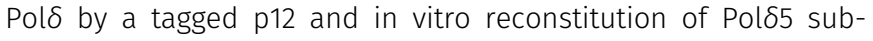
stantiates our prediction (Figs $2 \mathrm{~A}$ and 6 ). This is also supported by the fact that p12 was always present in higher stoichiometry in comparison with other subunits in Pol $\delta$ preparations by several other groups (Podust et al, 2002; Xie et al, 2002; Wang et al, 2011;
Zhou et al, 2011; Zhang et al, 2016; Lee et al, 2017). We also found that the dimerization of the fourth subunit of Pol $\delta$ is not restricted to human, as Cdm1 of SpPol $\delta$ also forms a dimer, which is again dependent on the KKR motif. As the RKR/KKR motif has been retained in other $\mathrm{p} 12$ homologues as well, it appears that such a property of the smallest subunit of Pol $\delta$ is evolutionarily conserved. Interestingly, the small accessory subunit of yet another B-family polymerase Pol $\zeta$, Rev7 is found to function as a dimer (Rizzo et al, 2018). Thus, the subunit dimerization of B-family DNA pols could be an intrinsic property, and it could be advantageous for the DNA polymerases to establish multiligand interactions during replication.

Most of the PCNA-interacting partners, including Pol $\delta$, bind to PCNA through a structurally conserved canonical PIP motif (Bruning \& Shamoo, 2004). Previously, we have shown that all the three subunits of ScPol $\delta$ contribute to PCNA interaction as well as its processive DNA synthesis (Acharya et al, 2011). The PIP motifs in Pol3, Pol31, and Pol32 have been mapped, and each of them can bind to a monomer of the trimeric PCNA. However, the scenario of hPol $\delta$ interacting to PCNA looks complex, and it is not clear which subunits primarily contribute to the PCNA interaction and the processivity of the enzyme. Although reports suggested that all the four subunits bind to PCNA and contribute enzymatic processivity to different degrees, identification of PIPs remains elusive (Lee et al, 2012). The PIP motif in p125 is yet to be identified. Like the Pol32 subunit, p68 possesses a conventional PIP motif at the extreme carboxyl terminal end (Bruning \& Shamoo, 2004), and accordingly,

Figure 7. Mutations in RKR motif inhibit binding with PCNA.

(A) The upper panel depicts Coomassie blue-stained gel of various p12 proteins resolved in a non-denaturing condition, whereas the lower panel is a far-Western analysis of a similar gel. Proteins were transferred from the gel to the membrane, and further, the blot was blocked with PCNA. After washings, the bound PCNA was detected by the anti-PCNA antibody. Lane 1: wild-type; lane 2: R3A, K4A, R5A; and lane 3: L104A, Y105A p12 proteins. (B) GST pull down of PCNA by wild-type p12. Beads of GST-p12 (lanes 1-3), GST-R3A, K4A, R5A (lanes 4-6), or GST-L104A, Y105A p12 mutants (lanes 7-9) were mixed with PCNA in equilibration buffer after the incubation beads were washed and the bound PCNA was eluted by the protein loading dye. Various fractions were resolved in 12\% SDS-PAGE, blotted to the membrane, and developed by the anti-PCNA antibody. Lanes 1, 4, and 7: 10\% of load; lanes 2, 5, and 8: 10\% of third washings; and lanes 3, 6, and 9: total eluates. (C) ITC analysis of binding of wild-type (i), R3A, K4A, R5A (ii), or L104A, Y105A mutant (iii) p12 to PCNA. In each panel, the upper half shows the measured heat exchanges during each PCNA protein injection. The lower half of each panel shows the enthalpic changes as a function of the molar ratio of the two proteins where p12 was considered as a dimer and PCNA as a trimer.

Source data are available for this figure. 
A

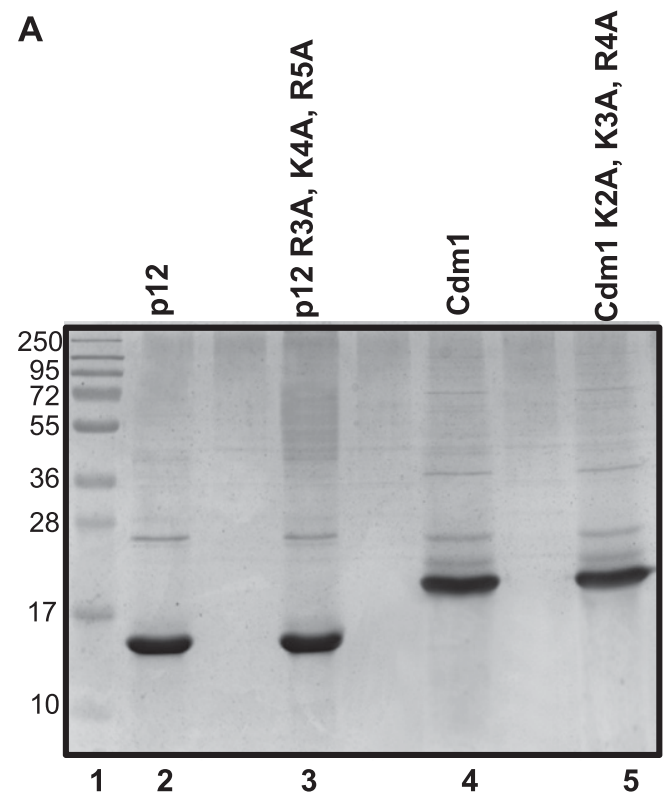

+ SDS
B

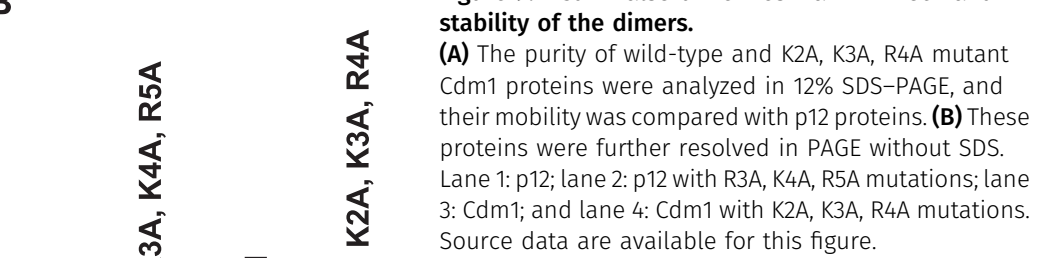

deletion of last 20 aa of p68 that encompass PIP sequences failed to bind to PCNA. Another study also revealed a mechanism that could modulate the interaction of p68 with PCNA by a protein kinasemediated phosphorylation of Ser458 in the PIP-box ${ }_{456}$ QVSITGFF $_{463}$ (Lemmens et al, 2008). Similarly, a 22-aa oligopeptide containing the PIP sequence ${ }_{57} \mathrm{LIQMRPF}_{64}$ of p50 was shown to bind PCNA by far-Western analysis (Lu et al, 2002; Wang et al, 2011); however, mutational analysis in this motif to provide functional evidence is yet to be carried out. Extensive biochemical studies carried out in the past suggest that the absence of p12 impedes processive DNA synthesis of Pol$\delta$. Here, by using structural modeling, yeast twohybrid system, and many other biophysical and chemical studies, we have mapped the PIP motif of p12 to be at the C-terminal tail that forms a typical $3_{10}$ helix and interacts with IDCL of PCNA. In addition, we showed that whereas the C-terminal sequence ${ }_{98} \mathrm{QCSLWHLY}_{105}$ is a PIP motif that is directly involved in PCNA interaction, the $\mathrm{N}$-terminal motif ${ }_{4} \mathrm{KRLITDSY}_{11}$ is involved in dimerization and that it indirectly participates in p12 interaction with PCNA. Contrary to an earlier observation, our study reveals that the role of the RKR motif in PCNA interaction is mostly indirect as the monomeric form of p12 does not bind to PCNA (Li et al, 2006).

There are sufficient pieces of evidence, both in vitro and in vivo, to support the idea that multiple subassemblies of Pol $\delta$ may exist. Proteolysis of p12 and p68 subunits by human calpain- 1 could trigger interconversion of Pol $\delta$ in the cell (Rahmeh et al, 2012; Terai et al, 2013; Zhou et al, 2012). Based on the dimerization of p12 and the purification of Pol $\delta 5$, we propose existence of four different

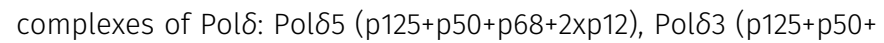
p68), Pol $\delta 2$ (p125+p50), and p125 alone (Fig 10A). Depending upon the cellular contexts such as either actively replicating cells or cells under genomic stress, this interconversion between the Pol $\delta$ s might happen. Pol $\delta 5$ could be the major holoenzyme that takes part in DNA replication, which was earlier extensively studied as Pol$\delta 4$. Our in vitro reconstitution assay rules out the purification of Pol $\delta 4$ with a monomeric p12; thus, it may not exist in the cell. So, now instead of four subunits, five subunits of Pol $\delta$ should be considered that will interact with the three available IDCLs in the trimeric PCNA, unless they use other binding sites such as inter-subunit junction or the C-terminal domain of PCNA (Eissenberg et al, 1997; Gomes \& Burgers, 2000). Genetic analyses of Pold PIPs in S. cerevisiae revealed that for cell survival, along with Pol32 PIP, any one among the Pol3 or Pol31 PIPs is essential. In the absence of functional Pol32 PIP domain, PIP domain mutation in Pol3 or Pol31 subunits causes lethality (Acharya et al, 2011). Despite being structural subunits, CDC27 and p68, the Pol32 homologues are essential in S. pombe and mice, respectively (Murga et al, 2016), and it explains their major role in Pol $\delta$ function in DNA synthesis. The binding affinity of Pol $\delta$ with PCNA also increases when p12 or p68 binds to the core $(K d=$ 8.7 9.3 $\mathrm{nM}$ ), and it further increases when all the subunits are present together $(K d=7.1 \mathrm{nM})$. The $K d$ of Pol $\delta$ core is found to be 73 nM (Lee et al, 2017). Accordingly, the addition of p68/Pol32 to the core (p125+p50 or Pol3+Pol31) results in high processivity; thus, its binding to PCNA appears to be critical. Considering all these, we propose a modified model for the network of protein-protein interactions of the Pol $\delta$-PCNA complex (Fig 10B). In a pentameric state of hPol $\delta$, along with p68, any other two subunits among p125/ p50/p12 will bind to PCNA in any combinations as shown in Fig 10B (i-iv). Upon p68 degradation or its phosphorylation, p125, p50, and one monomer of p12 dimer can bind to PCNA (v). Similarly, upon p12 proteosomal degradation as a response to DNA damage; the other three subunits will make contacts with PCNA (vi), whereas because of cleavage of both p68 and p12 in certain situations, p125 and p50 
A

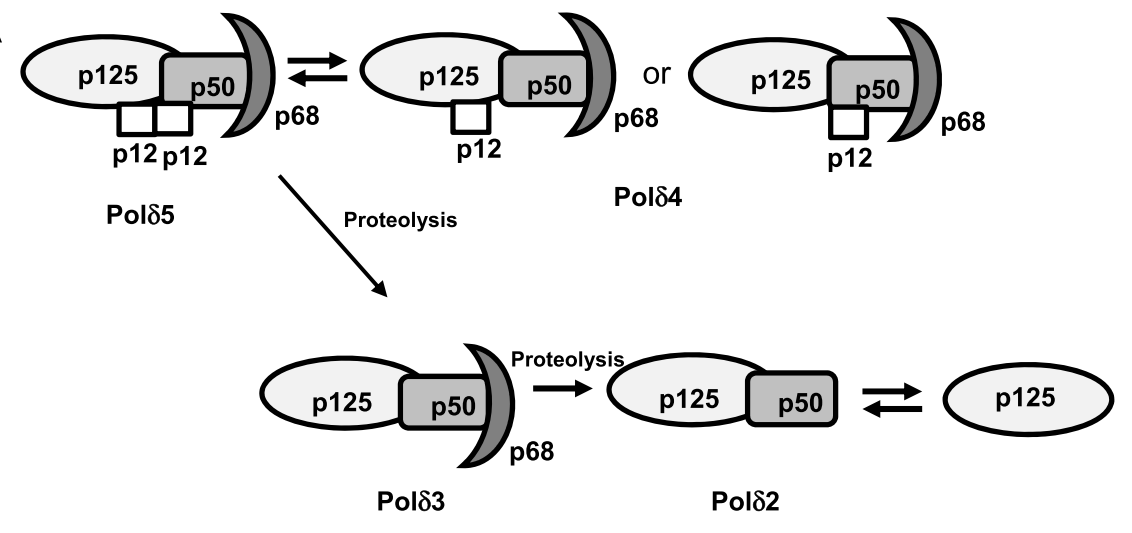

B
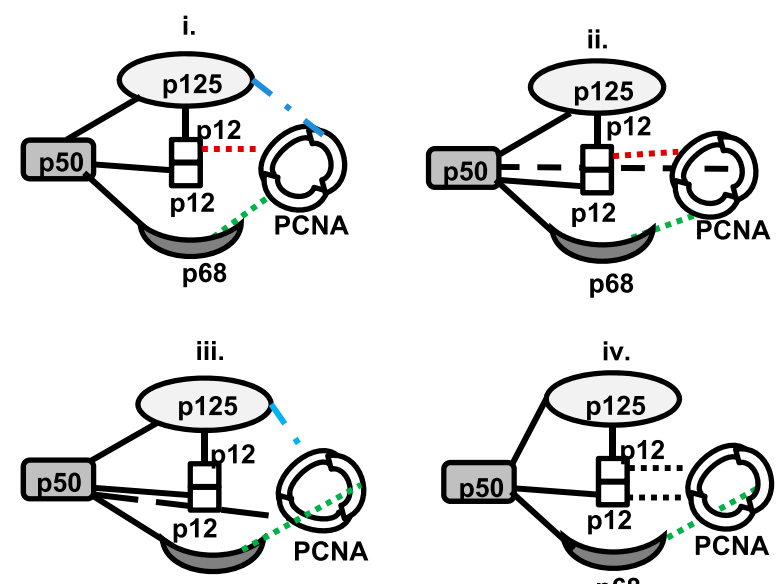

p68

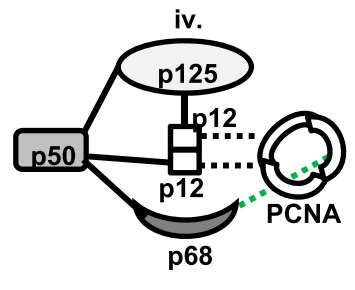

p68
Figure 10. Protein-protein network model for hPol $\delta$ and PCNA.

(A) Depicting different subassemblies of Pol$\delta$ complexes. As p12 is a dimer, pentameric Pol反 holoenzyme is proposed to function in DNA replication. Polס4 may or may not exist in the cell; however, after proteolysis during certain conditions such as genomic stress, Pol $\delta 5$ can be downgraded to Pol $\delta 3$ or Pol $\delta 2$ complexes. (B) Four different proposed modes of Pol $\delta 5$ binding to PCNA, where apart from p68, any other two subunits can bind to IDCLS of PCNA. In the dimerization state, only p12 can bind to PCNA (i-iv). Upon

phosphorylation of p68 or proteolysis of p12, other remaining three subunits bind to PCNA ( $v$ and vi). In the case of the core, both subunits interact with PCNA but with compromised processive DNA synthesis. PCNA monomers binding to p125, p50, p68, and p12 are shown in blue, black, red, and green dotted lines, respectively. Source data are available for this figure.
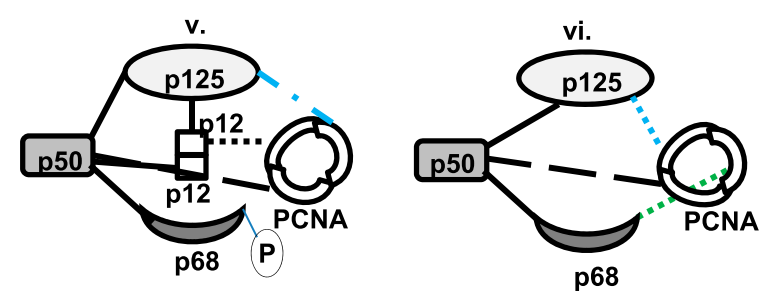

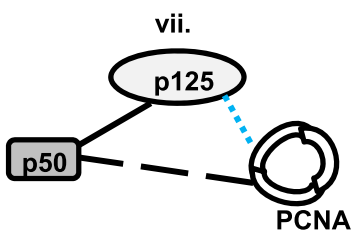

bind to PCNA (vii). Thus, this study warrants extensive mutational analyses as was carried out in ScPol$\delta$, rather than analyzing various sub-complexes to decipher the precise role of these PIPs in cellular function and processive DNA synthesis by $h$ Pol $\delta$.

In conclusion, here we show that RKR-mediated dimerization plays a vital role in 12 binding to PCNA and Pol $\delta 5$ architecture, and the phenomenon appears to be conserved throughout evolution.

\section{Materials and Methods}

Plasmids, oligonucleotides, antibodies, and enzymes

Human DNA polymerase $\delta$ constructs pET32-p125 and pCOLAhPold234 (a kind gift from Prof. Y Matsumoto) were used as precursor plasmids for subsequent manipulation (Schmitt et al, 2009). The oligonucleotides (Table S1) from Integrated DNA Technologies (IDT), Q5 high-fidelity DNA polymerase and other restriction enzymes from NEB, and antibodies from Sigma or Abcam were procured. Human PCNA was directly amplified from cDNA synthesized from total RNA of HELA cell line by using primers NAP239 and NAP240 and cloned into a pUC19 vector. The PCR product was digested with BamHI and cloned into the Bglll site to generate a bacterial expression system of GST-fused hPCNA. To express in budding yeast, hPCNA was amplified by using primers NAP251 and NAP240, the PCR product was digested with BamHI and cloned into the same site of pGBT9 and pGAD424 to generate Gal4BD-hPCNA and Gal4AD-hPCNA, respectively. Inverse PCR was carried out using the primer set NAP300-NAP304 on pUC19-hPCNA to generate pcna-79 (L126, 128AA), and further subcloned into an expression vector. Human pcna-90 (P253A, K254A) was PCR-amplified using primers 
NAP251 and NAP305 from the pUC19-hPCNA template; the PCR product was digested with $\mathrm{BamHI}$ and cloned into the same site of pUC19 and pGBT9. A GFP-fused hPCNA expression construct under the CMV promoter was a gift from Prof. Wim Vermeulen.

Amplified PCR products of wild type; R3A, K4A, R5A; and L104A, Y105A mutants of p12 from pCOLA-hPold234 by using primer pairs NAP261-NAP262, NAP362-NAP261, and NAP265-NAP262, respectively, were digested with EcoRI-BamHI and cloned into the same sites of pGBT9 and pGAD424. Similar PCR products amplified using the primers NAP260-NAP261, NAP373-NAP261, and NAP265-NAP260 were also digested with BamHI and cloned into the Bglll site of either pNA716 or p3X FLAG CMV7.1 vector to generate N-terminal GST-tag proteins expressed under $\mathrm{T7}$ promoter and the $\mathrm{N}$-terminal FLAG-tag human cell expression system, respectively. For confocal microscopy and pull-down studies, BamHI fragments of various p12 were subcloned into the BamHI site of the pcDNA-GFP vector. For RFP-fusion, the 12 fragment and the catalytic domain of Pol $\theta$ were amplified with the primer sets NAP448-NAP450 and NAP444-NAP151, respectively, digested with $\mathrm{EcoRI-BamHI}$, and cloned into the same sites of the pASred2-c1 vector.

Other hPolס subunits such as p125, p50, and p68 were also PCRamplified by the primer sets NAP252-NAP248, NAP254-NAP255, and NAP258-NAP257, respectively, digested with EcoRI-BamHI, and cloned into the same sites of pGBT9. Wild-type and K2A, K3A, R4A mutant of Cdm1 were PCR-amplified from S. pombe genomic DNA using primers NAP361-NAP451 and NAP361-NAP452, respectively, and the BamHI-digested products were cloned into the Bglll site in pNA716 for bacterial expression. Similarly, GST-hp125 expression plasmid was generated by cloning a BamHI-digested PCR product amplified from pET32-p125 as a template using NAP247 and NAP248 primers into the BgllI site of pNA716. All these constructs were authenticated by DNA sequencing.

\section{GST-fusion protein purification}

All GST-tagged proteins were expressed in Escherichia coli BL21 DE3 cells and purified by affinity chromatography using glutathionesepharose beads (GE Healthcare). The proteins were expressed as amino-terminal GST-fusion proteins under a T7 promoter. Briefly, 5$\mathrm{ml}$ pre-culture of the transformant was added to $500 \mathrm{ml} \mathrm{LB}+50 \mu \mathrm{g} /$ $\mathrm{ml}$ ampicillin and grown at $37^{\circ} \mathrm{C}$ till the $\mathrm{OD}_{600}$ reaches 0.6 . Next, the culture was induced with $1 \mathrm{mM}$ IPTG and allowed to grow for another $8 \mathrm{~h}$. Cells were harvested, and about $3 \mathrm{gm}$ of frozen cells were resuspended in 1× cell breaking buffer ( $50 \mathrm{mM}$ Tris- $\mathrm{HCl}, \mathrm{pH} 7.5,10 \%$ sucrose, $1 \mathrm{mM}$ EDTA, $500 \mathrm{mM} \mathrm{NaCl}, 0.5 \mathrm{mM}$ PMSF, $0.5 \mathrm{mM}$ benzamidine hydrochloride, $10 \mathrm{mM} \beta$-mercaptoethanol, and protease inhibitor cocktail). The cells were lysed with a high-pressure homogenizer at 10000 psi (Stansted). The lysate was cleared by centrifugation at $10000 \mathrm{rpm}$ for $10 \mathrm{~min}$. Furthermore, the supernatant was centrifuged at $30000 \mathrm{rpm}$ for $1 \mathrm{~h}$ in a P70AT rotor (Hitachi). Rest of the steps used for purification was the same as described before (Acharya et al, 2005). All the proteins were stored in the buffer containing a final concentration of $50 \mathrm{mM} \mathrm{Tris-HCl}(\mathrm{pH}$ 7.5), $150 \mathrm{mM} \mathrm{NaCl}, 10 \%$ glycerol, $5 \mathrm{mM} \mathrm{DTT}$, and $0.01 \% \mathrm{NP}-40$. The purity of the protein was confirmed after resolving on $12 \%$ SDSPAGE and stained by Coomassie blue.

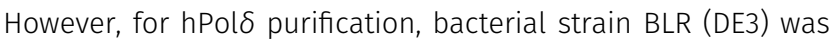
co-transformed with pLacRARE2 plasmid from Rosetta2 strain (Novagen), GST-p125, and pCOLA-hPold234; and colonies were selected on LB agar plates containing ampicillin $(50 \mu \mathrm{g} / \mathrm{ml})$, kanamycin $(30 \mu \mathrm{g} / \mathrm{ml})$, and chloramphenicol $(35 \mu \mathrm{g} / \mathrm{ml})$. About $60 \mathrm{ml}$ overnight-grown pre-culture was inoculated into 6 liters LB with mentioned antibiotics and grown at $37^{\circ} \mathrm{C}$ to an $\mathrm{OD}_{600}$ of 0.6 , followed by induction with $1 \mathrm{mM} \mathrm{IPTG}$, and further growth was continued for $15 \mathrm{~h}$ at $16^{\circ} \mathrm{C}$. The cells were harvested and stored at $-80^{\circ} \mathrm{C}$ until use. The cell breaking condition and other purification steps were followed as mentioned above. Taking advantage of the strategically located PreScission protease site, cleaved Pol84 (p125p50-p68-p12) was obtained in which only p12 subunit was aminoterminally FLAG-tagged. Similarly, p12 protein was also purified by using bacterial GST-p12 construct.

\section{Size-exclusion chromatography}

For size-exclusion chromatography, about $10 \mu \mathrm{g}$ of each freshly purified p12 protein was loaded onto a Superdex 200 PC3.2/30 minicolumn pre-equilibrated with a buffer containing $50 \mathrm{mM}$ Hepes $(\mathrm{pH}$ 7.5), $150 \mathrm{mM} \mathrm{NaCl}$, and 10\% glycerol. Chromatography was performed twice on an AKTA pure M system (GE Healthcare) at a flow rate of $0.05 \mathrm{ml} / \mathrm{min}$ at $4^{\circ} \mathrm{C}$, and the absorbance was monitored at $280 \mathrm{~nm}$

\section{Purification of human Pol $\delta 5$ complex}

To purify Pol $\delta 5$ complex; pre-purified Pol $\delta 4(10 \mu \mathrm{g})$ was mixed with an equal amount of untagged 12 protein under rocking conditions for $4 \mathrm{~h}$ at $4^{\circ} \mathrm{C}$. Then, the mixture was injected into a Superdex 200 PC3.2/30 minicolumn pre-equilibrated with a buffer containing $50 \mathrm{mM}$ Hepes (pH 7.5), $150 \mathrm{mM} \mathrm{NaCl}$, and 10\% glycerol. Chromatography was performed on an AKTA pure $\mathrm{M}$ system (GE Healthcare) at a flow rate of $0.03 \mathrm{ml} / \mathrm{min}$ at $4^{\circ} \mathrm{C}$, and the absorbance was monitored at $280 \mathrm{~nm}$. The eluate was collected in a 96-well plate fraction collector and then the various fractions were subjected to SDS-PAGE and Western blot analysis for the detection of individual subunits of hPold. The experiment was repeated thrice for Coomassie blue staining, but we could not detect any protein in the fractions. However, our Western analysis consistently reproduced the same results. To check the presence of Pol $\delta$ fractionation, the membrane was first probed with an antip68 antibody (Cat. No. WH0010714M1) because it does not directly interact with $\mathrm{p} 12$ and will give a clear indication of the complex. Furthermore, the enriched fractions were analyzed by probing with a specific antibody such as anti-p125 (Cat. No. SAB4200053), anti-p50 (Cat. No. SAB4200054), and anti-p12 (Cat. No. WH0057804M1). Horseradish peroxidase-conjugated host-specific secondary IgG (Cat. No. A90376154; Sigma-Aldrich and Cat. No. W402B; Abcam) was used to develop the blot by Chemidoc.

\section{Yeast two-hybrid analyses}

The yeast two-hybrid analyses were performed using HIS3 as a reporter system (Acharya et al, 2005). The HFY7C yeast strain (from Clonetech) was transformed with various combinations of the 
GAL4-AD (TRP1) and GAL4-BD (LEU2) fusion constructs. Cotransformants were obtained on synthetic dropout (SD) media plates lacking leucine and tryptophan. To verify the interaction, the transformants were grown on $5 \mathrm{ml}$ YPD liquid medium overnight at $30^{\circ} \mathrm{C}$ and various dilutions were either streaked or spotted on $\mathrm{Leu}^{-} \mathrm{Trp}^{-} \mathrm{His}^{-}$selection medium. Furthermore, the plates were incubated for $2 \mathrm{~d}$ at $30^{\circ} \mathrm{C}$ and photographed. Yeast transformants exhibiting histidine prototrophy are indicative of protein-protein interaction. The auxotrophic reporter assay was carried out by taking three different co-transformants.

\section{Formaldehyde cross-linking}

About 1-5 $\mu \mathrm{g}$ of native or mutant p12 protein in $20 \mathrm{mM}$ Hepes buffer (pH 7.5) was mixed with $0.5 \%$ formaldehyde solution for $30 \mathrm{~min}$ at $25^{\circ} \mathrm{C}$. The reaction was terminated by the addition of SDS sample buffer. Cross-linked proteins were resolved by electrophoresis in a $12 \%$ SDS-PAGE. The gel was stained with Coomassie blue. The experiment was repeated again with a different batch of purified proteins.

\section{Native PAGE analysis}

Wild-type and various mutants of $\mathrm{p} 12$ and Cdm1 proteins were mixed with a DNA loading dye and were analyzed on $12 \%$ native PAGE. The gel was run at $80 \mathrm{~V}$ for $5 \mathrm{~h}$ at $4^{\circ} \mathrm{C}$ using a running buffer containing $25 \mathrm{mM}$ Tris base and $192 \mathrm{mM}$ glycine, at $\mathrm{pH}$ 8.8. The proteins were visualized by Coomassie blue staining of the gels.

\section{Confocal microscopy}

CHO cells were grown up to $70 \%$ confluency on the cover glass in DMEM supplemented with $10 \%$ FBS and $1 \times$ penicillin-streptomycin antibiotics solution. Furthermore, the cells were washed with DPBS ( $\mathrm{pH} 7.4)$ and then replaced with DMEM containing 5\% FBS. These cells were co-transfected with GFP/RFP fusion constructs of $p 12$, PCNA, and a catalytic domain of Pol $\theta$ in various combinations as required by using the Lipofectamine Transfection kit as per the manufacturer's protocol (Invitrogen). Furthermore, the cells were incubated at $37^{\circ} \mathrm{C}$ with $5 \% \mathrm{CO}_{2}$ and $95 \%$ relative humidity. After $48 \mathrm{~h}$, the cells were thoroughly washed thrice with DPBS, fixed with icecold $100 \%$ methanol at $-20^{\circ} \mathrm{C}$ for $20 \mathrm{~min}$, followed by rinsing with DPBS ( $\mathrm{pH} 7.4$ ), and then the slides were prepared using antifade as a mounting agent. Images were taken using Leica TCS SP5 at 63x objective. Three independent experiments were carried out for each co-transfectant.

\section{Co-immunoprecipitation}

HEK293 cells were grown up to $70 \%$ confluency in a $10-\mathrm{cm}$ dish containing DMEM supplemented with $10 \%$ FBS and $1 \times$ penicillinstreptomycin antibiotics. These cells were co-transfected with FLAG-p12 with either GFP-p12 or GFP-p12 R3A, K4A, R5A mutant by using the Lipofectamine Transfection kit. The cells were grown in a humidified $\mathrm{CO}_{2}$ incubator at $37^{\circ} \mathrm{C}$. After $48 \mathrm{~h}$ of growth, the cells were harvested, washed thrice with DPBS, and immediately resuspended in RIPA buffer ( $50 \mathrm{mM}$ Tris- $\mathrm{HCl}, \mathrm{pH}$ 8.0, 0.5\% sodium deoxycholate, 1,000 mM NaCl, 0.1\% SDS, 1 mM EDTA, 1 mM EGTA, 25 mM sodium pyrophosphate, $1 \mathrm{mM} \beta$-glycerophosphate, $1 \mathrm{mM}$ sodium orthovanadate, and protease inhibitor tablet) and kept for $1 \mathrm{~h}$ at $4^{\circ} \mathrm{C}$ rocking condition. After centrifugation at 10,000 rpm, the supernatant was collected and protein concentration was determined using the Bradford method. About $500 \mu \mathrm{g}$ of total protein was incubated overnight with anti-FLAG antibody-conjugated agarose beads. The beads were washed thrice with RIPA buffer, and bound proteins were eluted by $40 \mu$ l of SDS loading buffer and subjected to $12 \%$ SDS-PAGE. The proteins from the gel were transferred to PVDF membrane, followed by incubation of the membrane with $5 \%$ skim milk in PBST for $1 \mathrm{~h}$ at room temperature. The blot was washed thrice with PBST and incubated with the anti-GFP antibody (1:5,000 dilution, Cat. No. ab290; Abcam) for 2 h at RT. Subsequently, after thorough washings, horseradish peroxidase-conjugated goat antirabbit IgG (diluted 1:10,000 in PBST, Cat. No. A6154; Sigma-Aldrich) was used to develop the blot.

Similarly, native hPol $\delta$ was co-immunoprecipitated from HEK293 cells transfected with GFP-p12. About $500 \mu \mathrm{g}$ of total protein was incubated overnight with anti-GFP or anti-p125 antibody-conjugated agarose beads. The beads were washed thrice with RIPA buffer, and bound proteins were eluted by $40 \mu \mathrm{l}$ of SDS loading buffer and subjected to 12\% SDS-PAGE. The proteins from the gel were transferred to PVDF membrane, cut into four pieces as per the molecular weight markers, and the membranes were individually incubated with $5 \%$ skim milk in PBST for $1 \mathrm{~h}$ at room temperature. The blot was washed thrice with PBST and probed with subunit-specific antibody for $2 \mathrm{~h}$ at room temperature. For p50 probing, first the membrane was probed with the anti-p50 antibody, then stripped off, and again probed with anti-GFP antibody as both the proteins migrate close to each other. Subsequently, after thorough washings, horseradish peroxidase-conjugated goat anti-IgG (diluted 1:10,000 in PBST, Cat. No. A6154; Sigma-Aldrich) was used to develop the blot.

\section{PCNA overlay assay}

Various proteins were resolved in two 12\% native PAGE, and whereas one of the gel developed with Coomassie blue, the other one was transferred to methanol-activated PVDF membrane. The blot was first washed with BLOTTO (25 mM Tris- $\mathrm{HCl}, \mathrm{pH} 7.4,150 \mathrm{mM}$ $\mathrm{NaCl}, 5 \mathrm{mM} \mathrm{KCl}, 5 \%$ fat-free milk, $1 \% \mathrm{BSA}$, and $0.05 \%$ Tween 20) for $1 \mathrm{~h}$ at room temperature. Then, the blot was incubated overnight at $4^{\circ} \mathrm{C}$ in $10 \mu \mathrm{g} / \mathrm{ml}$ of PCNA containing BLOTTO with constant agitation. After three rinses with BLOTTO, the membrane was incubated with the anti-PCNA antibody (diluted 1:1,000, Cat. No. SAB2108448; Sigma-Aldrich) in BLOTTO. Subsequently, after thorough washings, horseradish peroxidase-conjugated goat anti-rabbit IgG (diluted 1: 10,000 in PBST, Cat. No. A6154; Sigma-Aldrich) was used to develop the blot.

\section{GST pull-down assay}

GST wild-type or LY 104,105 AA p12 protein-bound glutathionesepharose beads were mixed with $0.5 \mu \mathrm{g}$ of either wild-type or 
mutant (L126A, I128A) human PCNA, and a pull-down experiment was carried out using a standardized protocol described previously (Acharya et al, 2005). Then the beads were thoroughly washed three times with 10 volumes of equilibration buffer $(50 \mathrm{mM}$ Tris$\mathrm{HCl}, \mathrm{pH} 7.5,150 \mathrm{mM} \mathrm{NaCl}, 5 \mathrm{mM}$ dithiothreitol, 0.01\% NP-40, and $10 \%$ glycerol). Finally, the bound proteins were eluted with $50 \mu \mathrm{l}$ SDS loading buffer. Various fractions were resolved on a $12 \%$ SDS-PAGE, followed by Western blot analysis similarly performed as in co-immunoprecipitation experiment except that the primary antibody used is an anti-PCNA antibody (Cat. No. SAB2108448; Sigma-Aldrich) in 1:750 dilutions.

\section{Isothermal titration calorimetry}

The purified $\mathrm{p} 12$ and PCNA proteins were dialyzed overnight in 1 liter of a buffer containing $20 \mathrm{mM}$ Hepes ( $\mathrm{pH} \mathrm{7.4)}$ and $150 \mathrm{mM} \mathrm{NaCl}$ at $4^{\circ} \mathrm{C}$ to ensure complete removal of DTT and glycerol from the protein storage buffer, which could affect the heat exchange. ITC assays were performed using a Malvern MicroCal PEAQ-ITC calorimeter. Before the experiment, the cell and the syringe were thoroughly washed with water, followed by cell rinsing with a buffer. A control run was carried out to make sure that the buffer is not participating in heat change where the cell was filled with $300 \mu \mathrm{l}$ of a buffer and concentrated $\mathrm{p} 12$ or PCNA protein $(120 \mu \mathrm{M})$ in the syringe. The titration did not show any false binding. ITC was performed using p12 $(10 \mu \mathrm{M})$ in the sample cell and PCNA or p12 $(120 \mu \mathrm{M})$ in the syringe. Twenty to twenty-five times $1.5-2 \mu \mathrm{l}$ of protein from the syringe was injected at intervals of $120 \mathrm{~s}$ with an initial delay of $120 \mathrm{~s}$ at $25^{\circ} \mathrm{C}$. For p12-p12 interaction, the reaction was carried out at $30^{\circ} \mathrm{C}$. Because of the binding of the ligand to protein in the cell, in these studies, the heat was generated and the difference of heat changes with respect to the reference cell that only contains water was detected and measured. The data were analyzed to determine various kinetic parameters using a single-site binding model provided in the ITC analysis software package. The experiments were repeated thrice with different batches of purified proteins.

\section{Circular dichroism}

The purified p12 proteins were dialyzed overnight into 1 liter of a buffer containing $20 \mathrm{mM}$ Hepes buffer ( $\mathrm{pH} 7.5$ ) and $20 \mathrm{mM} \mathrm{NaCl}$ at $4^{\circ} \mathrm{C}$. The secondary structure of $\mathrm{p} 12$ and $\mathrm{p} 12 \mathrm{R} 3 \mathrm{~A}, \mathrm{~K} 4 \mathrm{~A}, \mathrm{R} 5 \mathrm{~A}$ mutant was determined by $C D$ spectroscopy using a Chirascan (Applied Photophysics). Spectra were taken at $25^{\circ} \mathrm{C}$ in a $10-\mathrm{mm}$ path-length quartz cuvette containing the sample at concentrations of $0.2 \mathrm{mg} /$ $\mathrm{ml}$ of protein in $20 \mathrm{mM}$ Hepes buffer ( $\mathrm{pH} \mathrm{7.5)}$ and $20 \mathrm{mM} \mathrm{NaCl}$. The spectra were corrected for the buffer. Mean residue ellipticity values were calculated using the expression $[\theta]=\theta \times 100 /(\mathrm{cln})$, where $\theta$ is the ellipticity (in millidegrees), $c$ is the protein concentration (in mol/liter), l is the path length (in centimeter), and $\mathrm{n}$ is the number of amino acid residues. The analysis was repeated thrice with different batches of purified proteins.

\section{In silico analysis of p12 structures}

p12 RKR (1-MGRKRLITDSYPVK-14) and PIP (92-GDPRFQCSLWHLYPL-106) domains were used for peptide structure prediction by using
PEP-FOLD3 server (http://bioserv.rpbs.univ-paris-diderot.fr/services/ PEP-FOLD3/). The models generated were validated by the SAVES and Ramachandran plot. Furthermore, the generated structural models were aligned with PIP peptide sequences from p21 (1AXC) and p68 PIP (1U76).

\section{Supplementary Information}

Supplementary Information is available at https://doi.org/10.26508/lsa. 201900323.

\section{Acknowledgements}

We are grateful to Profs. Y Matsumoto and W Vermeulen for providing us hPol $\delta$ expression and CMV-GFP-hPCNA plasmids, respectively. We thank Sitendra Prasad Panda for his technical assistance, Dr. Jawed Alam for his involvement in initial studies, and Bhabani Shankar Sahoo for his help in confocal microscopy. Our laboratory colleagues are acknowledged for their thoughtful discussion. P Khandagale is a DBT senior research fellow; K Manohar and D Peroumal are thankful to CSIR-SRF and DBT-RA fellowships, respectively. This work was supported by the intramural core grant from Institute of Life Sciences, Bhubaneswar, India.

\section{Author Contributions}

P Khandagale: data curation, resources, formal analysis, validation, investigation, methodology, and writing-review and editing. D Peroumal: formal analysis, investigation, methodology, and writing-review and editing.

K Manohar: formal analysis, investigation, methodology, and writingreview and editing.

N Acharya: conceptualization, formal analysis, supervision, funding acquisition, validation, visualization, project administration, and writing-review and editing.

\section{Conflict of Interest Statement}

The authors declare that they have no conflict of interest.

\section{References}

Acharya N, Haracska L, Johnson RE, Unk I, Prakash S, Prakash L (2005) Complex formation of yeast Rev1 and Rev7 proteins: A novel role for the polymerase-associated domain. Mol Cell Biol 25: 9734-9740. doi:10.1128/mcb.25.21.9734-9740.2005

Acharya N, Klassen R, Johnson RE, Prakash L, Prakash S (2011) PCNA binding domains in all three subunits of yeast DNA polymerase delta modulate its function in DNA replication. Proc Natl Acad Sci USA 108: 17927-17932. doi:10.1073/pnas.1109981108

Albertson TM, Ogawa M, Bugni JM, Hays LE, Chen Y, Wang Y, Treuting PM, Heddle JA, Goldsby RE, Preston BD (2009) DNA polymerase epsilon and delta proofreading suppress discrete mutator and cancer phenotypes in mice. Proc Natl Acad Sci USA 106: 17101-17104. doi:10.1073/ pnas.0907147106 
Aria V, Yeeles JTP (2018) Mechanism of bidirectional leading-strand synthesis establishment at eukaryotic DNA replication origins. Mol Cell S10972765(18)30879-7. doi:10.1016/j.molcel.2018.10.019

Bermudez VP, MacNeill SA, Tappin I, Hurwitz J (2002) The influence of the Cdc27 subunit on the properties of the Schizosaccharomyces pombe DNA polymerase delta. J Biol Chem 277: 36853-36862. doi:10.1074/jbc. m202897200

Bruning JB, Shamoo Y (2004) Structural and thermodynamic analysis of human PCNA with peptides derived from DNA polymerase-delta p66 subunit and flap endonuclease-1. Structure 12: 2209-2219. doi:10.1016/ j.str.2004.09.018

Burgers PM (1998) Eukaryotic DNA polymerases in DNA replication and DNA repair. Chromosoma 107: 218-227. doi:10.1007/s004120050300

Burgers PMJ, Kunkel TA (2017) Eukaryotic DNA replication fork. Annu Rev Biochem 86: 417-438. doi:10.1146/annurev-biochem-061516-044709

Collins PE, Kiely PA, Carmody RJ (2014) Inhibition of transcription by B cell Leukemia 3 (Bcl-3) protein requires interaction with nuclear factor kappaB (NF-kappaB) p50. J Biol Chem 289: 7059-7067. doi:10.1074/jbc. $\mathrm{m} 114.551986$

Ducoux M, Urbach S, Baldacci G, Hubscher U, Koundrioukoff S, Christensen J, Hughes P (2001) Mediation of proliferating cell nuclear antigen (PCNA)-dependent DNA replication through a conserved p21(Cip1)like PCNA-binding motif present in the third subunit of human DNA polymerase delta. J Biol Chem 276: 49258-49266. doi:10.1074/jbc. m106990200

Eissenberg JC, Ayyagari R, Gomes XV, Burgers PM (1997) Mutations in yeast proliferating cell nuclear antigen define distinct sites for interaction with DNA polymerase delta and DNA polymerase epsilon. Mol Cell Biol 17: 6367-6378. doi:10.1128/mcb.17.11.6367

Essers J, Theil AF, Baldeyron C, van Cappellen WA, Houtsmuller AB, Kanaar R, Vermeulen W (2005) Nuclear dynamics of PCNA in DNA replication and repair. Mol Cell Biol 25: 9350-9359. doi:10.1128/mcb.25.21.9350-9359.2005

Fagerlund R, Melen K, Kinnunen L, Julkunen I (2002) Arginine/lysine-rich nuclear localization signals mediate interactions between dimeric STATs and importin alpha 5. J Biol Chem 277: 30072-30078. doi:10.1074 / jbc.m202943200

Fazlieva R, Spittle CS, Morrissey D, Hayashi H, Yan H, Matsumoto Y (2009) Proofreading exonuclease activity of human DNA polymerase delta and its effects on lesion-bypass DNA synthesis. Nucleic Acids Res 37: 2854-2866. doi:10.1093/nar/gkp155

Flohr T, Dai JC, Buttner J, Popanda O, Hagmuller E, Thielmann HW (1999) Detection of mutations in the DNA polymerase delta gene of human sporadic colorectal cancers and colon cancer cell lines. Int I Cancer 80: 919-929. doi:10.1002/(sici)1097-0215(19990315)80:6<919::aidijc19>3.0.co;2-u

Goldsby RE, Hays LE, Chen X, Olmsted EA, Slayton WB, Spangrude GJ, Preston BD (2002) High incidence of epithelial cancers in mice deficient for DNA polymerase delta proofreading. Proc Natl Acad Sci USA 99: 15560-15565. doi:10.1073/pnas.232340999

Gomes XV, Burgers PM (2000) Two modes of FEN1 binding to PCNA regulated by DNA. EMBO / 19: 3811-3821. doi:10.1093/emboj/19.14.3811

Haracska L, Acharya N, Unk I, Johnson RE, Hurwitz J, Prakash L, Prakash S (2005) A single domain in human DNA polymerase iota mediates interaction with PCNA: Implications for translesion DNA synthesis. Mol Cell Biol 25: 1183-1190. doi:10.1128/MCB.25.3.1183-1190.2005

Hindges R, Hubscher U (1997) DNA polymerase delta, an essential enzyme for DNA transactions. Biol Chem 378: 345-362.

Johansson E, Garg P, Burgers PM (2004) The Pol32 subunit of DNA polymerase delta contains separable domains for processive replication and proliferating cell nuclear antigen (PCNA) binding. J Biol Chem 279: 1907-1915. doi:10.1074/jbc.m310362200
Johnson RE, Klassen R, Prakash L, Prakash S (2015) A major role of DNA polymerase delta in replication of both the leading and lagging DNA strands. Mol Cell 59: 163-175. doi:10.1016/j.molcel.2015.05.038

Joiner WJ, Wang LY, Tang MD, Kaczmarek LK (1997) hSK4, a member of a novel subfamily of calcium-activated potassium channels. Proc Natl Acad Sci USA 94: 11013-11018. doi:10.1073/pnas.94.20.11013

Jones HM, Bailey MA, Baty CJ, Macgregor GG, Syme CA, Hamilton KL, Devor DC (2007) An NH2-terminal multi-basic RKR motif is required for the ATPdependent regulation of hIK1. Channels (Austin) 1: 80-91. doi:10.4161/ chan.3999

Krishna TS, Kong XP, Gary S, Burgers PM, Kuriyan I (1994) Crystal structure of the eukaryotic DNA polymerase processivity factor PCNA. Cell 79: 1233-1243. doi:10.1016/0092-8674(94)90014-0

Kunkel TA, Burgers PM (2014) Delivering nonidentical twins. Nat Struct Mol Biol 21: 649-651. doi:10.1038/nsmb.2852

Kunkel TA, Burgers PMJ (2017) Arranging eukaryotic nuclear DNA polymerases for replication: Specific interactions with accessory proteins arrange Pols alpha, delta, and in the replisome for leading-strand and lagging-strand DNA replication. Bioessays 39. doi:10.1002/ bies.201700070

Lee M, Wang X, Zhang S, Zhang Z, Lee EYC (2017) Regulation and modulation of human DNA polymerase delta activity and function. Genes (Basel) 8: E190. doi:10.3390/genes8070190

Lee MY, Zhang S, Lin SH, Chea J, Wang X, LeRoy C, Wong A, Zhang Z, Lee EY (2012) Regulation of human DNA polymerase delta in the cellular responses to DNA damage. Environ Mol mutagenesis 53: 683-698. doi:10.1002/em.21743

Lee MY, Zhang S, Lin SH, Wang X, Darzynkiewicz Z, Zhang Z, Lee EY (2014) The tail that wags the dog: 12 , the smallest subunit of DNA polymerase delta, is degraded by ubiquitin ligases in response to DNA damage and during cell cycle progression. Cell cycle 13: 23-31. doi:10.4161/ cc. 27407

Lemmens L, Urbach S, Prudent R, Cochet C, Baldacci G, Hughes P (2008) Phosphorylation of the $\mathrm{C}$ subunit (p66) of human DNA polymerase delta. Biochem biophysical Res Commun 367: 264-270. doi:10.1016/j. bbrc.2007.12.083

Li H, Xie B, Zhou Y, Rahmeh A, Trusa S, Zhang S, Gao Y, Lee EY, Lee MY (2006) Functional roles of $\mathrm{p} 12$, the fourth subunit of human DNA polymerase delta. J Biol Chem 281: 14748-14755. doi:10.1074/jbc.m600322200

Lu X, Tan CK, Zhou JQ, You M, Carastro LM, Downey KM, So AG (2002) Direct interaction of proliferating cell nuclear antigen with the small subunit of DNA polymerase delta. J Biol Chem 277: 24340-24345. doi:10.1074/ jbc.m200065200

Manohar K, Acharya N (2015) Characterization of proliferating cell nuclear antigen (PCNA) from pathogenic yeast Candida albicans and its functional analyses in S. Cerevisiae. BMC Microbiol 15: 257. doi:10.1186/ s12866-015-0582-6

Meng X, Zhou Y, Lee EY, Lee MY, Frick DN (2010) The p12 subunit of human polymerase delta modulates the rate and fidelity of DNA synthesis. Biochemistry 49: 3545-3554. doi:10.1021/bi100042b

Miyabe I, Kunkel TA, Carr AM (2011) The major roles of DNA polymerases epsilon and delta at the eukaryotic replication fork are evolutionarily conserved. PLoS Genet 7: e1002407. doi:10.1371/ journal.pgen.1002407

Murga M, Lecona E, Kamileri I, Diaz M, Lugli N, Sotiriou SK, Anton ME, Mendez J, Halazonetis TD, Fernandez-Capetillo O (2016) POLD3 is haploinsufficient for DNA replication in mice. Mol Cel 63: 877-883. doi:10.1016/j.molcel.2016.07.007

Pavlov YI, Shcherbakova PV, Rogozin IB (2006) Roles of DNA polymerases in replication, repair, and recombination in eukaryotes. Int Rev Cytol 255: 41-132. doi:10.1016/s0074-7696(06)55002-8 
Podust VN, Chang LS, Ott R, Dianov GL, Fanning E (2002) Reconstitution of human DNA polymerase delta using recombinant baculoviruses: The p12 subunit potentiates DNA polymerizing activity of the four-subunit enzyme. J Biol Chem 277: 3894-3901. doi:10.1074/jbc.m109684200

Pohler JR, Otterlei M, Warbrick E (2005) An in vivo analysis of the localisation and interactions of human p66 DNA polymerase delta subunit. BMC Mol Biol 6: 17. doi:10.1186/1471-2199-6-17

Rahmeh AA, Zhou Y, Xie B, Li H, Lee EY, Lee MY (2012) Phosphorylation of the p68 subunit of Pol delta acts as a molecular switch to regulate its interaction with PCNA. Biochemistry 51: 416-424. doi:10.1021/bi201638e

Rizzo AA, Vassel FM, Chatterjee N, D'Souza S, Li Y, Hao B, Hemann MT, Walker GC, Korzhnev DM (2018) Rev7 dimerization is important for assembly and function of the Rev1/Polzeta translesion synthesis complex. Proc Natl Acad Sci USA 115: E8191-E8200. doi:10.1073/pnas.1801149115

Schmitt MW, Matsumoto Y, Loeb LA (2009) High fidelity and lesion bypass capability of human DNA polymerase delta. Biochimie 91: 1163-1172. doi:10.1016/j.biochi.2009.06.007

Stillman B (2008) DNA polymerases at the replication fork in eukaryotes. Mol Cell 30: 259-260. doi:10.1016/j.molcel.2008.04.011

Tahirov TH, Makarova KS, Rogozin IB, Pavlov YI, Koonin EV (2009) Evolution of DNA polymerases: An inactivated polymerase-exonuclease module in Pol epsilon and a chimeric origin of eukaryotic polymerases from two classes of archaeal ancestors. Biol Direct 4: 11. doi:10.1186/1745-61504-11

Terai K, Shibata E, Abbas T, Dutta A (2013) Degradation of p12 subunit by CRL4Cdt2 E3 ligase inhibits fork progression after DNA damage. I Biol Chem 288: 30509-30514. doi:10.1074/jbc.c113.505586

van Hennik PB, ten Klooster JP, Halstead JR, Voermans C, Anthony EC, Divecha N, Hordijk PL (2003) The C-terminal domain of Rac1 contains two motifs that control targeting and signaling specificity. J Biol Chem 278: 39166-39175. doi:10.1074/jbc.m307001200

Waga S, Bauer G, Stillman B (1994) Reconstitution of complete SV40 DNA replication with purified replication factors. I Biol Chem 269: 10923-10934

Wang L, Zhang K, Wu L, Liu S, Zhang H, Zhou Q, Tong L, Sun F, Fan Z (2012) Structural insights into the substrate specificity of human granzyme $\mathrm{H}$ : The functional roles of a novel RKR motif. J Immunol 188: 765-773. doi:10.4049/jimmunol.1101381
Wang Y, Zhang Q, Chen H, Li X, Mai W, Chen K, Zhang S, Lee EY, Lee MY, Zhou Y (2011) P50, the small subunit of DNA polymerase delta, is required for mediation of the interaction of polymerase delta subassemblies with PCNA. PLoS One 6: e27092. doi:10.1371/journal.pone.0027092

Xie B, Mazloum N, Liu L, Rahmeh A, Li H, Lee MY (2002) Reconstitution and characterization of the human DNA polymerase delta four-subunit holoenzyme. Biochemistry 41: 13133-13142. doi:10.1021/bi0262707

Yoon JH, Acharya N, Park J, Basu D, Prakash S, Prakash L (2014) Identification of two functional PCNA-binding domains in human DNA polymerase $\mathrm{K}$. Genes Cells 19: 594-601. doi:10.1111/gtc.12156

Zerangue N, Schwappach B, Jan YN, Jan LY (1999) A new ER trafficking signal regulates the subunit stoichiometry of plasma membrane K(ATP) channels. Neuron 22: 537-548. doi:10.1016/s0896-6273(00)80708-4

Zhang P, Mo JY, Perez A, Leon A, Liu L, Mazloum N, Xu H, Lee MY (1999) Direct interaction of proliferating cell nuclear antigen with the p125 catalytic subunit of mammalian DNA polymerase delta. J Biol Chem 274: 26647-26653. doi:10.1074/jbc.274.38.26647

Zhang Q, Zhang Q, Chen H, Chen Y, Zhou Y (2016) Multiple forms of human DNA polymerase delta sub-assembling in cellular DNA transactions. Curr Protein Pept Sci 17: 746-755. doi:10.2174/ 1389203717666160226145006

Zhou Y, Chen H, Li X, Wang Y, Chen K, Zhang S, Meng X, Lee EY, Lee MY (2011) Production of recombinant human DNA polymerase delta in a Bombyx mori bioreactor. PLoS One 6: e22224. doi:10.1371/journal. pone.0022224

Zhou Y, Meng X, Zhang S, Lee EY, Lee MY (2012) Characterization of human DNA polymerase delta and its subassemblies reconstituted by expression in the MultiBac system. PLoS One 7: e39156. doi:10.1371/ journal.pone.0039156

Zuo S, Bermudez V, Zhang G, Kelman Z, Hurwitz I (2000) Structure and activity associated with multiple forms of Schizosaccharomyces pombe DNA polymerase delta. J Biol Chem 275: 5153-5162. doi:10.1074/jbc.275.7.5153

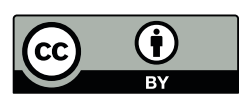

License: This article is available under a Creative Commons License (Attribution 4.0 International, as described at https://creativecommons.org/ licenses/by/4.0/). 